\title{
Molecular dynamics simulation study of deformation mechanisms in 3C-SiC during nanometric cutting at elevated temperatures
}

\author{
Saeed Zare Chavoshi, Xichun Luo* \\ Centre for Precision Manufacturing, Department of Design, Manufacture and Engineering \\ Management, University of Strathclyde, Glasgow, G1 1XJ, UK \\ *Corresponding author: xichun.luo@strath.ac.uk
}

\begin{abstract}
:
Molecular dynamics (MD) simulation was employed in this study to elucidate the dislocation/amorphization-based plasticity mechanisms in single crystal 3C-SiC during nanometric cutting on different crystallographic orientations across a range of cutting temperatures, $300 \mathrm{~K}$ to $3000 \mathrm{~K}$, using two sorts of interatomic potentials namely analytical bond order potential (ABOP) and Tersoff potential. Of particular interesting finding while cutting the $(110)<00 \overline{1}>$ was the formation and subsequent annihilation of stacking fault-couple and Lomer-Cottrell (L-C) lock at high temperatures, i.e. $2000 \mathrm{~K}$ and $3000 \mathrm{~K}$, and generation of the cross-junctions between pairs of counter stacking faults meditated by the gliding of Shockley partials at $3000 \mathrm{~K}$. Another point of interest was the directional dependency of the mode of nanoscale plasticity, i.e. while dislocation nucleation and stacking fault formation were observed to be dominant during cutting the $(110)<00 \overline{1}>$, low defect activity was witnessed for the $(010)<100>$ and $(111)<\overline{1} 10>$ crystal setups. Nonetheless, the initial response of $3 \mathrm{C}-\mathrm{SiC}$ substrate was found to be solid-state amorphization for all the studied cases. Further analysis through virtual X-ray diffraction (XRD) and radial distribution function (RDF) showed the crystal quality and structural changes of the substrate
\end{abstract}


during nanometric cutting. A key observation was that the von Mises stress to cause yielding was reduced by $49 \%$ on the (110) crystal plane at $3000 \mathrm{~K}$ compared to what it took to cut at $300 \mathrm{~K}$. The simulation results were supplemented by additional calculations of mechanical properties, generalized stacking faults energy (GSFE) surfaces and ideal shear stresses for the two main slip systems of 3C-SiC given by the employed interatomic potentials.

Keywords: Molecular dynamics; 3C-SiC; Plasticity; Defect formation; Amorphization; Nanometric cutting

\section{Introduction}

Due to its exceptional physical and chemical properties, silicon carbide ( $\mathrm{SiC}$ ) has become a consummate candidate for the fabrication of nano-devices which require high performance in extreme environments. 3C-SiC, as a zinc blende structured $\mathrm{SiC}$ possesses the highest fracture toughness and hardness among the SiC polytypes. It is a kind of brittle material at room temperature owing to its relatively low fracture toughness and high hardness, i.e. 2.02 $\mathrm{MPa} \mathrm{m}^{1 / 2}$ and $26.4 \mathrm{GPa}$ [1], respectively. This restricted room-temperature plasticity makes this material to show poor machinability. It is believed that with the increase of temperature, plasticity plays a greater role in the fracture and deformation processes of $3 \mathrm{C}-\mathrm{SiC}$ primarily as a result of thermally-generated intrinsic defects and thermal softening processes.

Appreciation of crystal plasticity of materials subjected to complex loading at low and high temperatures has been a long-lasting challenge in materials science. There are few studies on discovering the plastic deformation mechanisms of 3C-SiC under a variety of different contact loading conditions such as pressure loading [2-3], nanoscratching [4-5], nanoindentation [6-9] and nanometric cutting [10-11]. What is known from these studies is that there is substantial incongruence on the plasticity mechanisms of 3C-SiC. For instance, while shear instability induced 
amorphization was considered by Tang [4] as the underlying mechanism in nanoscratching, Noreyan et al. [5] attributed the plastic behaviour to the rocksalt structural transformation of 3CSiC. Similarly, Goel et al. [10] claimed that 3C-SiC undergoes $s p^{3}-s p^{2}$ disorder during nanometric cutting whereas Mishra and Szlufarska [11] attributed the plasticity to the dislocation nucleation event. However, the extant literature concerning nanoscale plasticity of $3 \mathrm{C}-\mathrm{SiC}$ is seen to focus on study of the deformation behaviour at room temperature $(300 \mathrm{~K})$ and there exists no research on revealing the mechanisms involved in the plasticity of this material at elevated temperatures. Particularly, in nanometric cutting which is a shear-dominant process, resulting in more deviatoric stress conditions thus multiple plasticity mechanisms are conceivable; hence providing an extensive insight of the plastic deformation of 3C-SiC. Accordingly, the following four important research questions regarding the plasticity of $3 \mathrm{C}-\mathrm{SiC}$ during high temperature nanometric cutting were recognized and attempted to be answered in this study:

1- How do the thermally-driven phenomena contribute to the crystal plasticity of 3C-SiC during nanometric cutting at elevated temperatures?

2- What is the role of amorphization in driving the plasticity of $3 \mathrm{C}-\mathrm{SiC}$ ?

3- What is the critical magnitude of the stress causing single crystal 3C-SiC to flow during its nanometric cutting? More particularly, how do the critical yield stresses e.g. von Mises stress, octahedral stress, Tresca stress and Principal stresses vary during elevated temperature deformation?

4- How much influence does the employed interatomic potential on the aforementioned parameters?

In order to answer these questions, this work used molecular dynamics (MD) simulation to explore incipient plasticity and mechanisms involved in the plastic deformation of single crystal $3 \mathrm{C}-\mathrm{SiC}$ during nanometric cutting on the (010), (110) and (111) crystallographic orientations at a wide range of temperatures $(300 \mathrm{~K}-3000 \mathrm{~K})$ using two types of interatomic potentials namely analytical 
bond order potential (ABOP) [12] and Tersoff potential [13]. In order to evaluate the plasticity mechanisms occurring in the hot nanometric cutting, dislocation extraction algorithm (DXA) [14] was adopted and the generalized stacking faults energy (GSFE) surfaces and ideal shear stresses were calculated for the two main slip systems of 3C-SiC in order to obtain a better appreciation of defect formation. The local environment of atoms up to the second neighbour shell was also monitored and virtual $\mathrm{XRD}$ along with RDF analysis was performed to qualify the structural changes during nanometric cutting.

\section{Modelling and simulation methodology}

\subsection{Nanometric cutting simulation model}

Inspired from the previous works of the authors [15-18], the same simulation model was adapted in this study, which is shown in Fig. 1. Similar to those studies, the diamond cutting tool in this work was modelled as a deformable body. The substrate and the cutting tool were divided into three distinct areas namely, Newtonian, thermostat and boundary zones. Atoms in the boundary region were kept fixed so as to reduce boundary effect and maintain lattice regularity. The simulation model is extremely small and hence the thermostat area was used to artificially disperse the extra heat generated within the cutting zone which in reality would have otherwise been taken away by chips and coolant. Newtonian area follows traditional Newtonian mechanics (LAMMPS NVE dynamics) so that the employed potential energy function decides the position of atoms as they are integrated over time. Periodic boundary condition (PBC) was applied along the $z$ direction. In order to address the problem of lattice mismatch of silicon and diamond in periodic boundary condition, the periodic box dimension was selected so that the two lattice constants were in an integer proportion. Further description on the adjustment procedure concerning mismatch in the lattice constant between silicon and diamond while employing PBC can be found elsewhere [19]. To keep the simulation model simple, a major assumption here was to use the vacuum environment. This 
consideration although discards the role of oxidation of $3 \mathrm{C}$-SiC which is a practical reality but at the same time, it provides a healthy data to compare, contrast and highlight purely the role of temperature in influencing the deformation behaviour of 3C-SiC.

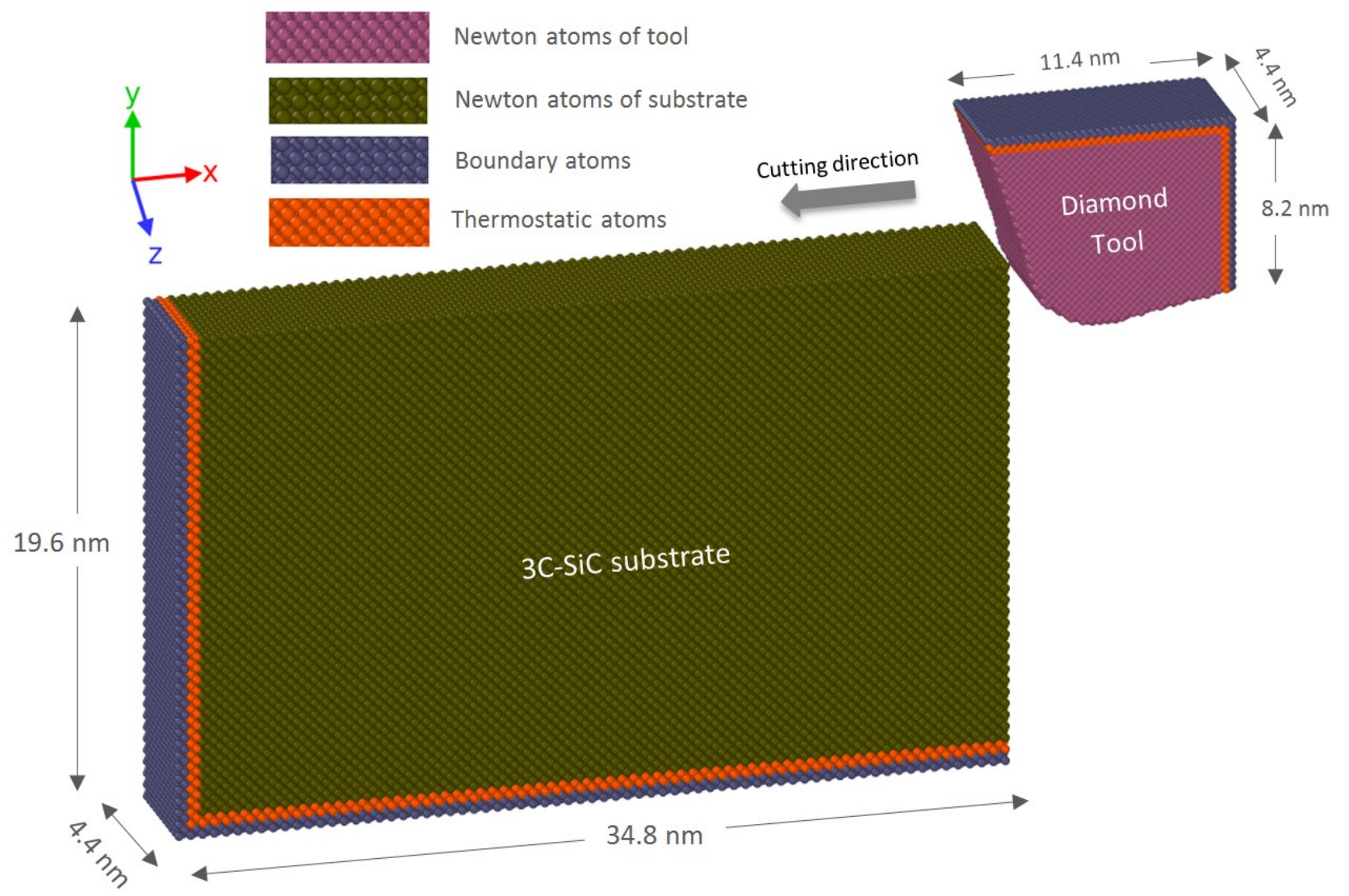

Fig 1. Schematic model of the nanometric cutting simulation

\subsection{Interatomic potential energy function}

Development of potential energy function is a fertile area in the MD simulation arena. For this reason, various potential functions have been developed to describe the interaction between constituent atoms. Since the accuracy and reliability of classical MD simulations is governed by the potential function, precautions are needed while selecting interatomic potential for a specific system and process. Pastewka et al. [20] recently highlighted some key considerations required to employ a 
potential energy function to model the phenomena of fracture, wear or plasticity in materials such as silicon, carbon and silicon carbide $(\mathrm{SiC})$. The most broadly adopted formalism for simulating Si-C system is the Tersoff potential function [13], which was developed in 1994 to appropriately estimate the ground-state structure, energies, and elastic properties of both $3 \mathrm{C}-\mathrm{SiC}$ and amorphous $\mathrm{SiC}$. One decade later, Erhart and Albe [12] proposed an analytical bond order potential (ABOP) which could describe both bulk as well as the dimer properties of $\mathrm{SiC}$ more properly over Tersoff potential. Therefore, in this study, both the ABOP and Tersoff potentials were utilized to define the interactions between the atoms of silicon and carbon and to make a comparison between the results.

\subsection{Simulation procedure}

MD simulations were implemented by using a public-domain computer code, known as "large-scale atomic/molecular massively parallel simulator" (LAMMPS) [21]. The simulated cutting temperatures were $300 \mathrm{~K}, 900 \mathrm{~K}, 1200 \mathrm{~K}, 1400 \mathrm{~K}, 2000 \mathrm{~K}$ and $3000 \mathrm{~K}$, regulated via a Berendsen thermostat. The details of simulation model and the process parameters employed in the simulations are shown in Table 1.

Using improper equilibrium lattice constant will force the simulation system far away from equilibrium and consequently the accuracy of the MD simulation results is inaccurate. Hence, the simulation model was fed with the appropriate lattice constants shown in Table 2, which were calculated as the equilibrium lattice constants at various temperatures from the potentials employed in this study. The equilibrium lattice constant of diamond (cutting tool) at $300 \mathrm{~K}$ was computed as $3.568 \AA$ and $3.558 \AA$ for the $\mathrm{ABOP}$ and Tersoff potentials, respectively. At the start of the simulation, the diamond tool was set at a distance of $10 \AA(1 \mathrm{~nm})$ from the $3 \mathrm{C}$-SiC substrate (free travel before cutting), in order to ensure that the tool atoms are far from the equilibrium cut-off range of the Si-C interaction potential. The simulation model was equilibrated for a total time of 30 ps before the commencement of cutting. The trials were performed at a cutting speed of $50 \mathrm{~m} / \mathrm{s}$ 
since it is almost impossible to implement parametric investigations at real cutting speeds, which are anticipated to be about 1 to $2 \mathrm{~m} / \mathrm{s}$. The visualization of atomic trajectories was carried out using “Open Visualization Tool" (OVITO) [22] and "Dislocation Extraction Algorithm" (DXA) [14] was employed for the sake of analysing dislocation and defects.

Table 1. Details of the MD simulation model and the cutting parameters used in the study

\begin{tabular}{|c|c|}
\hline Substrate material & Single crystal $3 \mathrm{C}-\mathrm{SiC}$ \\
\hline Substrate dimensions & $34.8 \times 19.6 \times 4.4 \mathrm{~nm}^{3}$ \\
\hline Tool material & Single crystal diamond \\
\hline Cutting edge radius (tip radius) & $3.5 \mathrm{~nm}$ \\
\hline Uncut chip thickness (cutting depth in 2D) & $3 \mathrm{~nm}$ \\
\hline Cutting orientation and cutting direction & $\begin{array}{l}\text { Case } 1:(010)<100> \\
\text { Case } 2:(110)<00 \overline{1}> \\
\text { Case } 3:(111)<\overline{1} 10>\end{array}$ \\
\hline $\begin{array}{l}\text { Rake and clearance angle of the cutting } \\
\text { tool }\end{array}$ & $-25^{\circ}$ and $10^{\circ}$ \\
\hline Substrate temperature & $\begin{array}{c}300 \mathrm{~K}, 900 \mathrm{~K}, 1200 \mathrm{~K}, 1400 \mathrm{~K}, 2000 \mathrm{~K} \text { and } \\
3000 \mathrm{~K}\end{array}$ \\
\hline Cutting speed & $50 \mathrm{~m} / \mathrm{s}$ \\
\hline Time step & $1 \mathrm{fs}$ \\
\hline Potential energy function & ABOP [12] and Tersoff [13] \\
\hline
\end{tabular}

Table 2. Calculated equilibrium lattice constants and cohesive energy of single crystal 3C-SiC at different temperatures using ABOP and Tersoff potential energy functions

\begin{tabular}{|c|c|c|c|c|c|}
\hline Temperature $(\mathrm{K})$ & $\begin{array}{c}\text { ABOP } \\
\text { lattice } \\
\text { constant }(\AA)\end{array}$ & $\begin{array}{c}\text { ABOP } \\
\text { cohesive } \\
\text { energy }(\mathrm{eV})\end{array}$ & $\begin{array}{c}\text { Tersoff } \\
\text { lattice } \\
\text { constant } \\
(\AA)\end{array}$ & $\begin{array}{c}\text { Tersoff } \\
\text { cohesive } \\
\text { energy }(\mathrm{eV})\end{array}$ & $\begin{array}{c}\text { Experimental } \\
\text { lattice constant } \\
(\AA)[23]\end{array}$ \\
\hline
\end{tabular}




\begin{tabular}{|c|c|c|c|c|c|}
\hline 300 & 4.3623 & -6.3376 & 4.3113 & -6.4005 & 4.3581 \\
\hline 900 & 4.3681 & -6.3342 & 4.3534 & -6.3269 & 4.3689 \\
\hline 1200 & 4.3712 & -6.3325 & 4.3703 & -6.2878 & 4.3754 \\
\hline 1400 & 4.3738 & -6.3297 & 4.3806 & -6.26088 & 4.3798 \\
\hline 2000 & 4.4409 & -6.1033 & 4.4109 & -6.17797 & - \\
\hline 3000 & 4.4838 & -5.9718 & 4.452 & -6.04078 & - \\
\hline
\end{tabular}

\section{Results and discussion}

\subsection{Assessment of accuracy of the interatomic potential functions}

The accuracy of the employed potential functions in reproducing of elastic constants and other mechanical characteristics was assessed and summarized in Table 3. In general, ABOP potential is more precise particularly in producing the Voigt averages, which are known to be better to deal with local strains around dislocations [24].

Table 3. Material properties of single crystal 3C-SiC obtained by experiment and MD simulations.

\begin{tabular}{|c|c|c|c|}
\hline Properties of 3C-SiC & ABOP & Tersoff & Exp. \\
\hline $\mathrm{C}_{11}(\mathrm{GPa})$ & 382 & 447 & $390[25]$ \\
\hline $\mathrm{C}_{12}(\mathrm{GPa})$ & 145 & 134 & $142[25]$ \\
\hline $\mathrm{C}_{44}(\mathrm{GPa})$ & 240 & 293 & $256[25]$ \\
\hline Anisotropy ratio $(\mathrm{A})$ & 1.98 & 1.87 & 2.06 \\
\hline$C_{11}-C_{12}$ & & & \\
\hline Anisotropy factor $(\mathrm{H})(\mathrm{GPa})$ & 243 & 273 & 264 \\
$2 C_{44}+C_{12}-C_{11}$ & & & \\
\hline Bulk modulus $(\mathrm{B})(\mathrm{GPa})$ & & & \\
$C_{11}+2 C_{12}$ & 224 & 241 & 225 \\
\hline 3 & & & \\
\hline Young's modulus $(\mathrm{E} 100)(\mathrm{GPa})$ & 302 & 385 & 314 \\
\hline
\end{tabular}




\begin{tabular}{|c|c|c|c|}
\hline$C_{11}-2 \frac{C_{12}}{C_{11}+C_{12}} C_{12}$ & & & \\
\hline $\begin{array}{l}\left.\text { Young's modulus ( } \mathrm{E}_{110}\right)(\mathrm{GPa}) \\
4 \frac{\left(C_{11}{ }^{2}+C_{12} C_{11}-2 C_{12}{ }^{2}\right) C_{44}}{2 C_{44} C_{11}+C_{11}{ }^{2}+C_{12} C_{11}-2 C_{12}{ }^{2}}\end{array}$ & 446 & 540 & 467 \\
\hline $\begin{array}{l}\text { Young's modulus }\left(\mathrm{E}_{111}\right)(\mathrm{GPa}) \\
\qquad 3 \frac{C_{44}\left(C_{11}+2 C_{12}\right)}{C_{11}+2 C_{12}+C_{44}}\end{array}$ & 531 & 623 & 557 \\
\hline $\begin{array}{l}\text { Voigt shear modulus }(\mu)(\mathrm{GPa}) \\
\qquad C_{44}-\frac{1}{5} H\end{array}$ & 191.4 & 238.4 & 203.2 \\
\hline $\begin{array}{l}\text { Voigt Poisson's ratio }(v) \\
\frac{C_{12}-\frac{H}{5}}{2\left(C_{12}+C_{44}-2 \frac{H}{5}\right)}\end{array}$ & 0.167 & 0.125 & 0.152 \\
\hline $\begin{array}{l}\text { Voigt Young's modulus (E) (GPa) } \\
\qquad 2 \mu(1+v)\end{array}$ & 446.7 & 536.4 & 468.2 \\
\hline
\end{tabular}

In order to obtain a better understanding of defect formation, the generalized stacking fault energy (GSFE) surfaces and ideal shear stresses required to nucleate dislocations for the two main slip systems of the zinc blende structure of $3 \mathrm{C}-\mathrm{SiC}$, shuffle $<110>\{111\}$ and glide $<112>\{111\}$, were assessed. 3C-SiC structure is defined as the stacking of double-layers $\{111\}$ planes. The space between two successive double-layers is known as the shuffle set, while the space between the two planes of the double-layer is called glide set. The lowest energy barrier required to be crossed for the slip from the ideal configuration to the intrinsic stacking fault configuration is called unstable GSFE surfaces, which is improbable to be measured experimentally [26]. The unstable GSFE 
surfaces can be combined with Peierls-Nabarro (PN) models [27-28] to estimate dislocation core properties. Fig. $2 \mathrm{a}$ and $2 \mathrm{~b}$ depict the GSFE curves obtained via block shearing process. Two interatomic potentials exhibit almost similar qualitative behaviour for the shuffle and glide sets. The unstable and intrinsic stacking fault configurations correspond to a slip of $0.342 a_{0}$ and $0.409 a_{0}\left(a_{0}\right.$ is the lattice constant), respectively, in the $<112>$ directions. The projection of GSFE curves on the $<110>$ direction is symmetric corresponding to a slip of $0.353 a_{0}$. As seen in Fig. 2 a, the value of unstable stacking fault energy $\left(\gamma_{u s}\right)$ in the glide $<112>\{111\}$ predicted by the ABOP is lower than that of the Tersoff potential, indicative of smaller energy barrier, suggesting that dislocations can be nucleated more easily while using the ABOP potential. It is worth pointing out here that the DFT calculates the unstable GSFE surfaces in the glide set to be $0.1747 \mathrm{eV} / \AA^{2}$ [29-30], suggesting the overestimation of the unstable GSFE by the two empirical potentials employed in this study. Nevertheless, the given value by the ABOP is closer to the DFT calculation. Furthermore, the intrinsic stacking fault energy $\left(\gamma_{i s f}\right)$ for Tersoff is found much higher than that of ABOP, indicating that the stacking faults are less likely to form while applying Tersoff potential. It is generalized that stacking faults are common in SiC owing to the low stacking fault energy [31]. The ab initio calculations revealed a value of $\gamma_{i s f}=0.2323 \mathrm{eV} / \AA^{2}[32]$, which is close to the magnitude given by the $\operatorname{ABOP}\left(0.2717 \mathrm{eV} / \AA^{2}\right)$.

It is also instructive to mention that the unstable twinning fault energy $\left(\gamma_{u t}\right)$ estimated by the ABOP is higher than that of the Tersoff. The high ratio of $\gamma_{u t} / \gamma_{u s}(\sim 1.58)$ for the ABOP shows that the energy barriers for twinning formation are higher than those for dislocation nucleation thus twinning is difficult to form (low twinability) while utilizing this potential. However, this value for the Tersoff is close to unity, signifying the ease of twinning formation under specific circumstances. As for the shuffle $<110>\{111\}$ slip, the predicted unstable GSFE by the Tersoff potential is lower than that of the ABOP. The calculated unstable GSFE surface in the shuffle set by the DFT is 0.169 $\mathrm{eV} / \AA^{2}$ [29-30], which is much lower than those estimated by the two potential functions. It is of 
note that the dislocation activity is strongly subject to the crystallographic orientations of cutting plane and cutting direction [33].

In order to obtain better insights into the favourable slip systems in our simulation setup, the gradient of GSFE surfaces for the shuffle and glide slip systems is plotted in Fig. 2c-d. In these plots, the maximum magnitude corresponds to the ideal shear stress $\left(\tau_{0}\right)$ needed for destabilizing a perfect crystal sheared on the shuffle $<110>\{111\}$ or glide $<112>\{111\}$. Hence, $\tau_{0}$ reflects the critical stress required to nucleate a dislocation from a free surface in a pure crystal. As observed in Fig. $2 \mathrm{c}-\mathrm{d}$, the ideal shear stress related to the nucleation of a dislocation on the glide set is $\sim 35 \mathrm{GPa}$ and $\sim 40 \mathrm{GPa}$, respectively, for the ABOP and Tersoff potentials. It appears that the ideal shear stress in the glide set is overpredicted by both the potentials in comparison with the DFT calculations, which is $\sim 28 \mathrm{GPa}$ [34]. On the shuffle set, the ABOP potential predicts the ideal shear stress to be $\sim 55 \mathrm{GPa}$ whereas the corresponding value estimated by the Tersoff is $\sim 48 \mathrm{GPa}$ at a shorter Burgers vector. The ideal shear stress for the shuffle set calculated by DFT is $~ 37 \mathrm{GPa}$ [34]. It can be inferred that the ideal shear strength of the glide $<112>\{111\}$ slip systems are lower than that of the shuffle $<110>\{111\}$ slip systems, signifying that the glide $<112>\{111\}$ slip systems are more favourable than shuffle $<110>\{111\}$ slip systems. More importantly, the Schmid factor of glide slip system is 0.47 which is larger than that of shuffle slip system, i.e. 0.41. In addition, glide dislocations are more sensitive to thermal activation [35].

Overall, it can conclude that $\mathrm{ABOP}$ is more robust in predicting the mechanical properties especially Voigt averages which are associated to the dislocations. Besides, dislocation nucleation in the glide set and stacking fault formation given by ABOP are in better agreement with the DFT and $a b$ initio calculations. Hence, we report the results based on the ABOP function and whenever needed, comparison is made with the results obtained by Tersoff potential. 

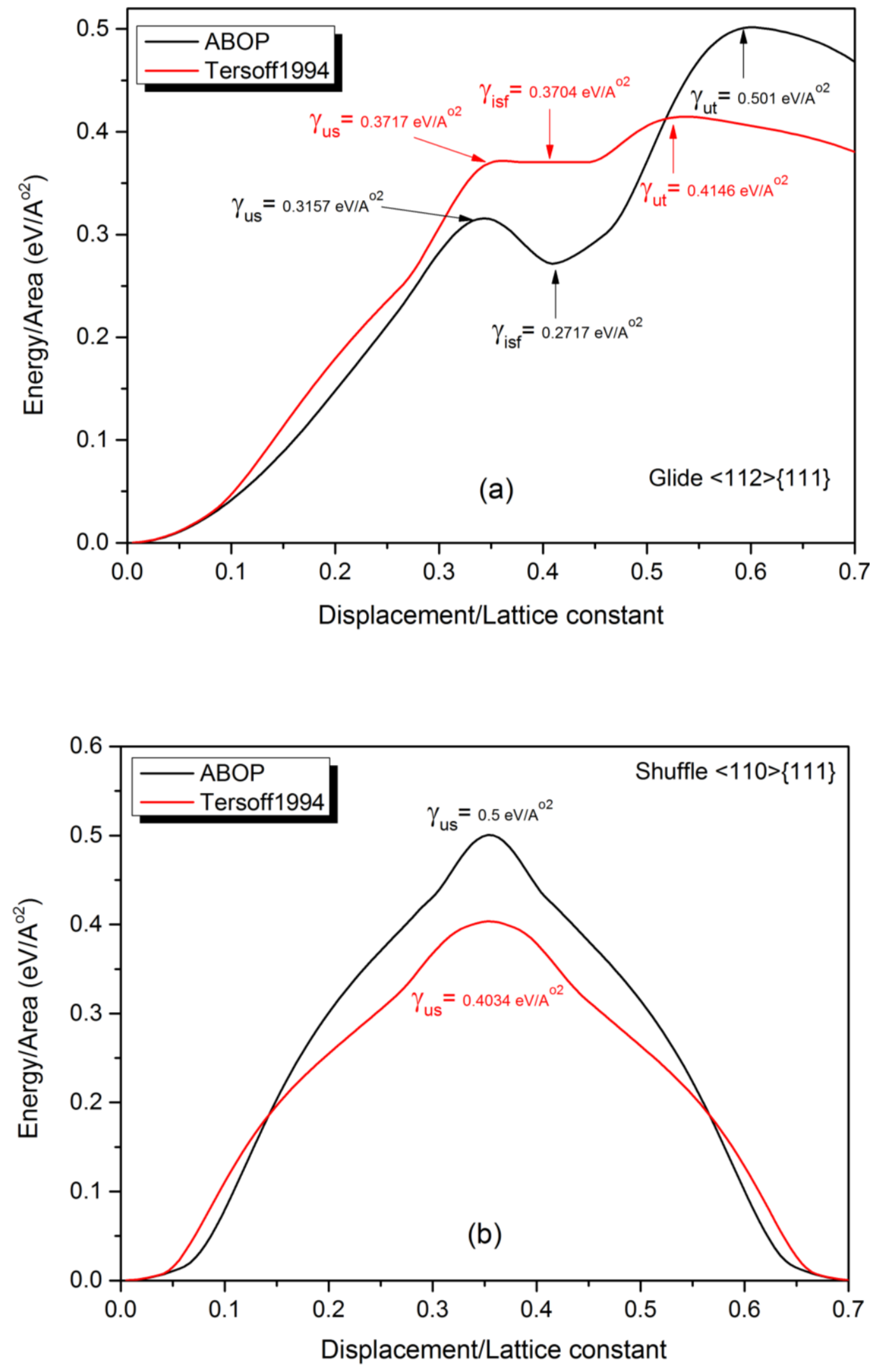

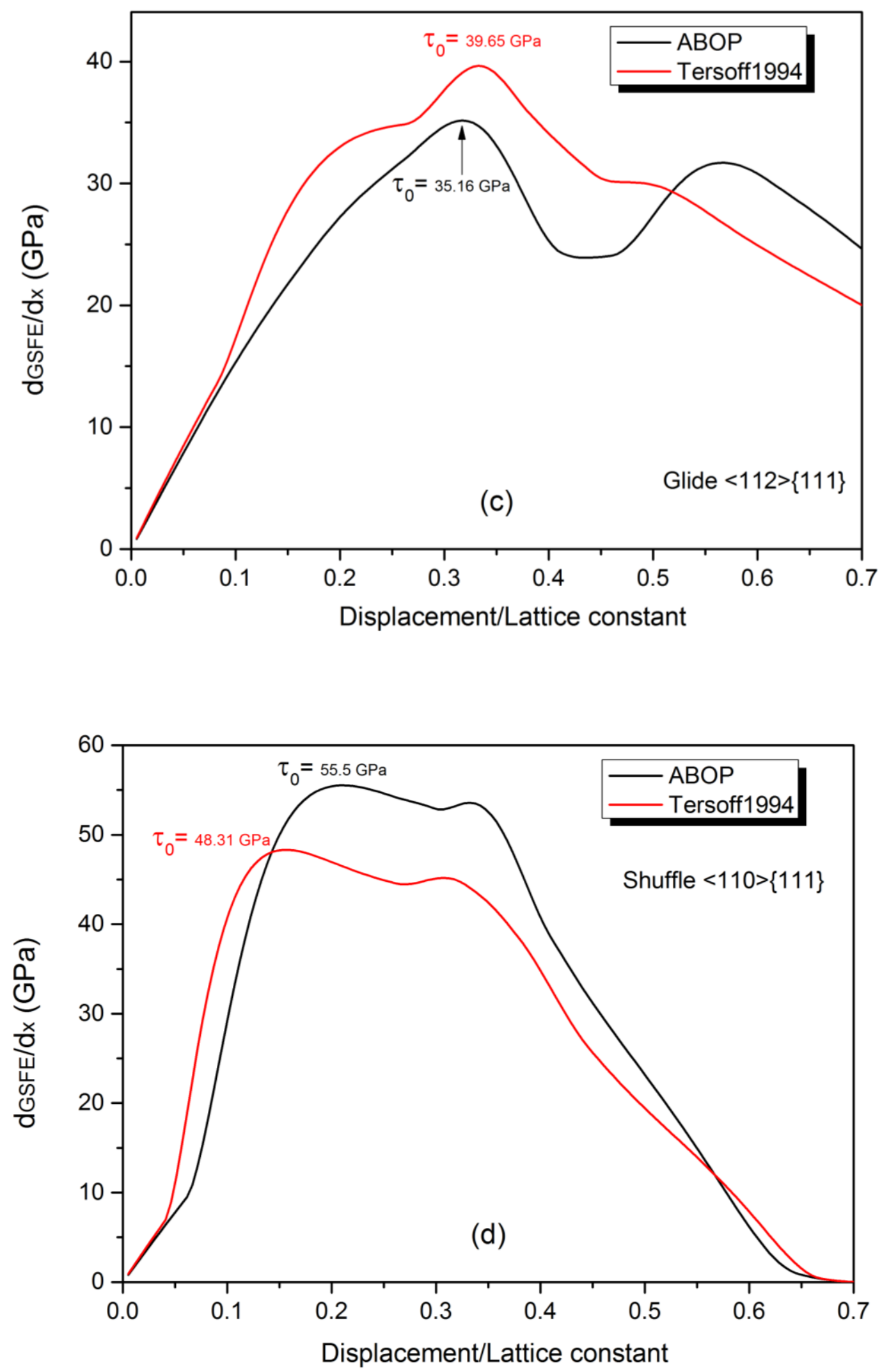

Fig. 2. GSFE curves and ideal shear stress $\left(\tau_{0}\right)$ for the glide $<112>\{111\}$ and shuffle $<110>\{111\}$ slip systems obtained by the ABOP and Tersoff interatomic potentials 


\subsection{Analysis of crystal defects}

Single crystal 3C-SiC is regarded as a face-centred cubic (FCC) crystal in the Bravais lattice group, which simply results in multiple-slips on the four equivalent $\{111\}$ planes. The preferred slip systems are closely correlated to the core structure of their mobile dislocations. Hence, dislocations have to glide in these planes with a trivial lattice resistance, as estimated by P-N models [27-28]. As a starting point, we analyse the crystal defect formation when cutting is performed on the (110) orientation since this cutting direction and cutting plane is more prone to defect formation according to the simulation setup. Fig. 3a-b demonstrates the crystal defects at a cutting distance of $10 \mathrm{~nm}$ when nanometric cutting is implemented at $300 \mathrm{~K}$. The perfect dislocation loop with $1 / 2<110>$ Burgers vectors is seen to glide ahead of the tool tip. V-shaped perfect dislocation and partial half loop are also nucleated ahead of the tool, facilitating the plasticity of 3C-SiC. Such V-shaped dislocations were also seen by Mishra and Szlufarska [33] during sliding/cutting at a cutting depth of $3.5 \mathrm{~nm}$. Additionally, it is generalized that the non-equilibrium intrinsic point defects such as vacancies can be created during plastic deformation process [36], leading to the formation of the vacancy-originated stacking fault in the Si-C monolayer. So, the stacking fault observed in Fig. 3b could be a consequence of formation of such point defects. These observations confirm that aside from formation of one-dimensional defects, two-dimensional defects, i.e. stacking faults, can be generated within the restricted volume of $3 \mathrm{C}-\mathrm{SiC}$ substrate during nanometric cutting at room temperature. Stacking fault formation in $3 \mathrm{C}-\mathrm{SiC}$ was also observed in multimillion-atom $\mathrm{MD}$ simulation of nanoindentation [37] and in thick 3C-SiC crystals grown by CF-PVT [38]. Note that the white atoms shown in Fig. 3 represent the highly-discorded (amorphous) atoms, which have been obtained through monitoring the local environment of atoms up to the second neighbour shell. In Fig. 3, the chip and subsurface of substrate attain amorphous state, signifying order-disorder (amorphization) transition of 3C-SiC atoms. The results are in accord with the simulations of Goel et al. [39], which showed that atoms in the chip and subsurface of the substrate lose their pristine diamond cubic lattice. Indeed, the initial response of substrate was observed to be the formation of 
amorphous atoms followed by the defect formation. In section 3.3, amorphization is discussed in detail. Of interest is that Mishra and Szlufarska [40] reported that dislocation activity can lead to amorphization near the surface of $3 \mathrm{C}-\mathrm{SiC}$ during nanoindentation, and connected their observations to the amorphous chips observed in nanomachining experiments on $6 \mathrm{H}-\mathrm{SiC}$ [41]. However, dislocation-induced amorphization was not observed in our nanometric cutting simulations.

It can be seen from Fig. $3 \mathrm{c}$ that with the progress of cutting, the stacking faults are decomposed and Shockley partials with $1 / 6<112>$ Burgers vectors are formed, gliding on the closely packed $\{111\}$ planes, which are the glide planes of a zinc blende crystal, accompanied by the perfect dislocations. Some dislocation interactions and junctions were also observed in both cases, viz. at $10 \mathrm{~nm}$ and $20 \mathrm{~nm}$ cutting distance. Furthermore, the DXA analysis reveals some dislocations, which cannot be regarded as physical dislocations and they are merely artefacts since no continuous chain of core atoms along the dislocation line was observed in Fig. 3.

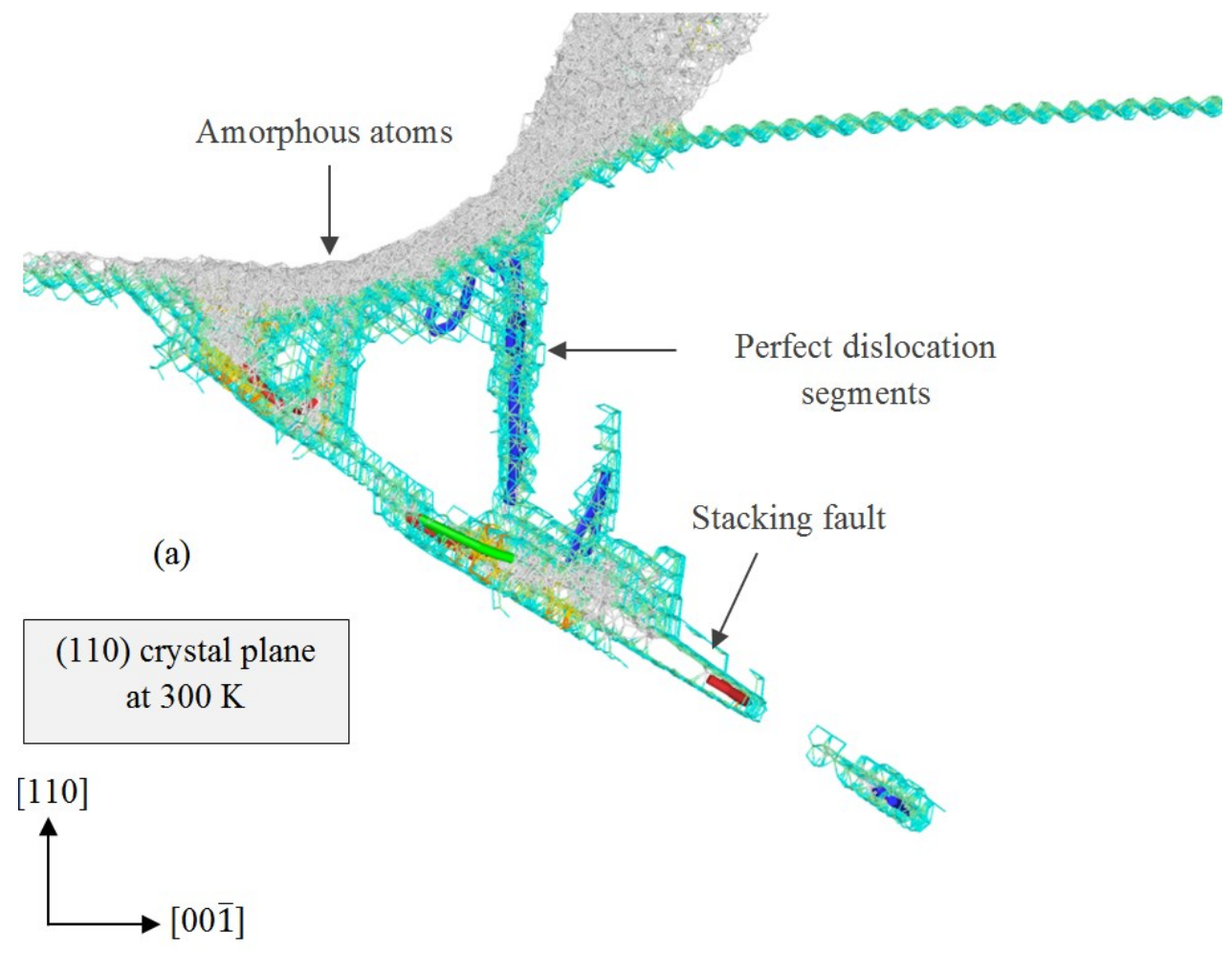



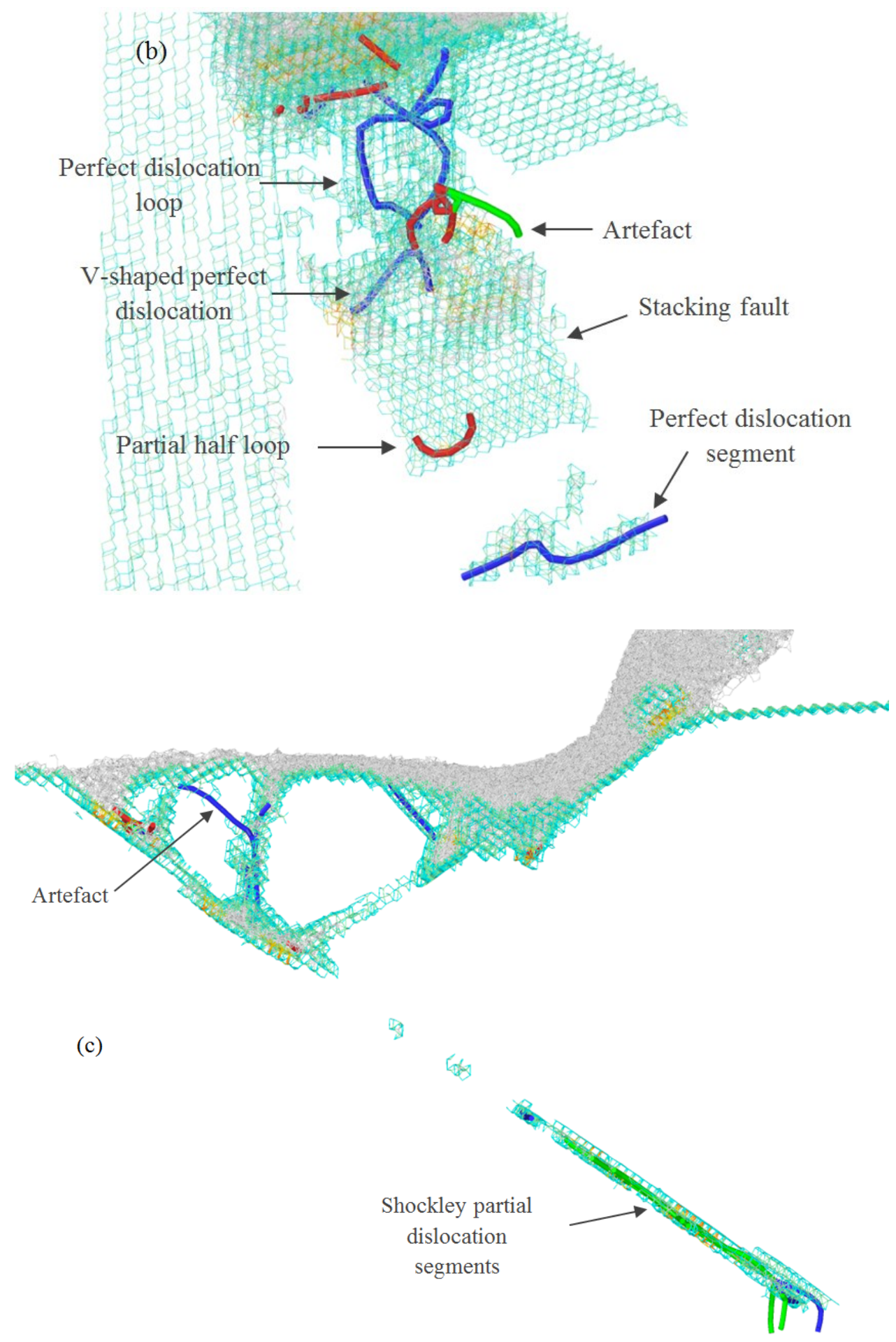

Fig. 3. MD simulation output illustrating amorphous atoms (white atoms), dislocation nucleation and formation of stacking faults while cutting the (110) plane at $300 \mathrm{~K}$ at a cutting distance of $\mathrm{a}, \mathrm{b}$ ) $10 \mathrm{~nm} \mathrm{c)} 20 \mathrm{~nm}$. Blue, red and green rods, respectively, stand for the perfect, Shockley partial and unknown partial dislocations.

During deformation processes, it is anticipated that increase of the temperature leads to an increase in the energy available for thermal activation; hence the deformation rate enhances [42]. In general, the thermal fluctuations facilitate to overcome the energy barrier for dislocation nucleation. As seen 
in Fig. 4a, more dislocations are nucleated when nanometric cutting is performed at $900 \mathrm{~K}$. The total length of dislocations nucleated within the $3 \mathrm{C}-\mathrm{SiC}$ substrate is $\sim 2.5$ times higher than that of 300 K. Shockley partial segments, loop and half loop are observed to nucleate. Furthermore, a stacking fault sandwiched between two Shockley partials is formed. Indeed, the glide of this dislocation-originated stacking fault is meditated by the Shockley partials through dislocationstacking fault interaction. Of interest is the nucleation of long perfect dislocations within the substrate. At $20 \mathrm{~nm}$ cutting distance, dislocations are further propagated within the substrate which improves the crystal plasticity of 3C-SiC. Dislocation multijunction and dissociation of perfect dislocations into Shockley partials and stair-rod partials with $1 / 6<110>$ Burgers vectors can be witnessed in Fig. 4b. Interestingly, Frank-type sessile partials with $1 / 3<111>$ Burgers vectors were observed to nucleate, joint with the perfect dislocation and Shockley partials. It is informative to mention that the partial dislocations in $3 \mathrm{C}-\mathrm{SiC}$ are assumed to be terminated by only one element, either Si-core or C-core. The Si-core dislocation nucleation is more energy-favourable whereas the C-core dislocation is more likely to nucleate under higher temperatures [43]. 

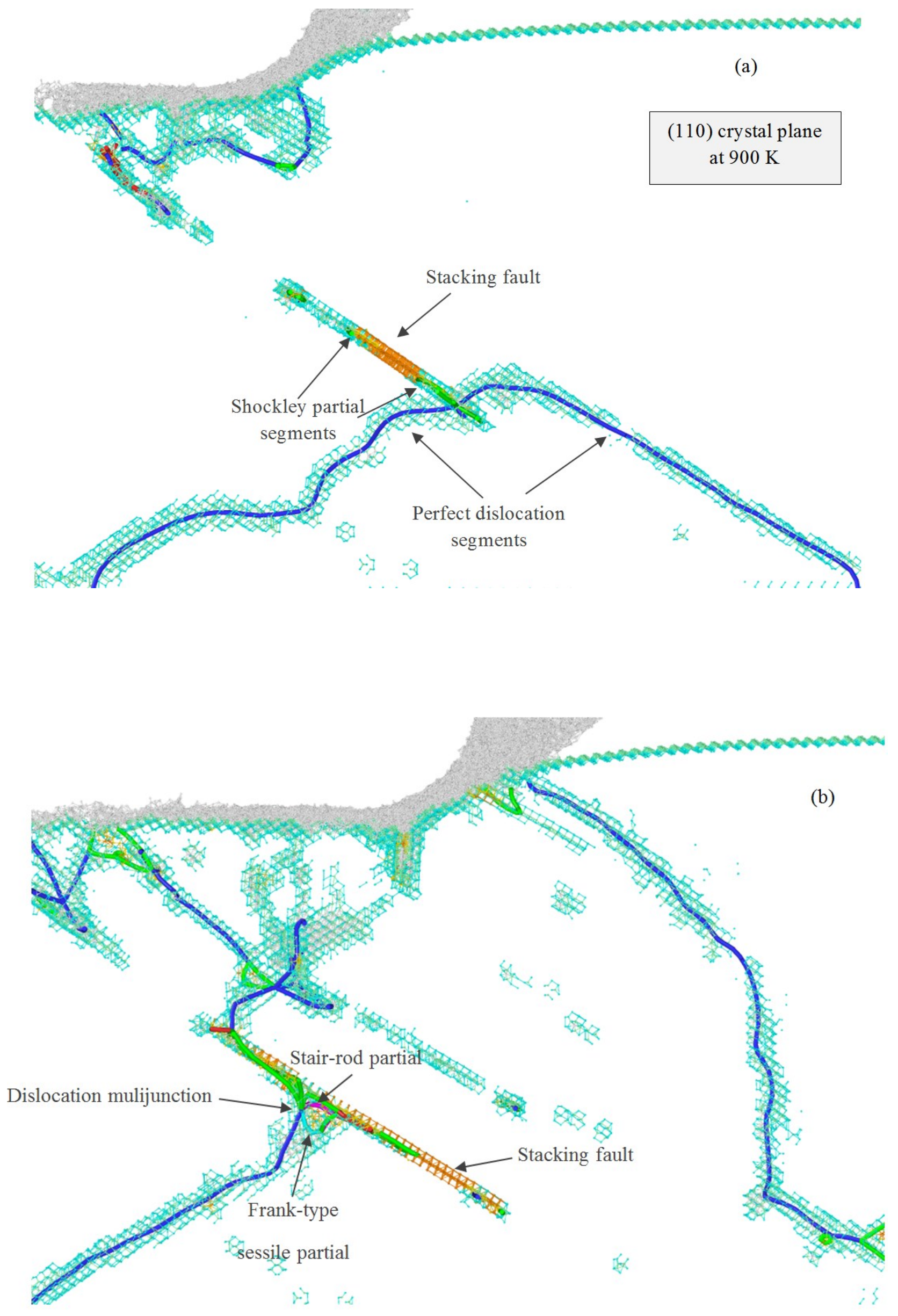
Fig. 4. Formation of crystal defects while cutting the (110) plane at $900 \mathrm{~K}$ at a cutting distance of a) $10 \mathrm{~nm} \mathrm{b)} 20 \mathrm{~nm}$. Pink and aqua rods represent the stair-rod and Frank partial dislocations.

Fig. 5a demonstrates that the defect formation mode at $1200 \mathrm{~K}$ is relatively similar to that of $900 \mathrm{~K}$. However, longer perfect dislocation ahead of the tool and a stacking fault beneath the tool tip can be seen to form in this case. Phonon and thermal vibration of the atoms within the substrate rises due to the thermal excitation, leading to an increase in the probability of having atoms with higher energy state in the system. When the thermal energy is adequately high, there exists the possibility of having an atom jump out of the lattice, creating a point defect in the material. The generated point defect can lead to the formation of the vacancy-originated stacking fault. Hence, the stacking fault observed underneath the tool tip in Fig. 5a could be a result of a point defect. Shockley partial loops and dislocation-originated stacking fault are also nucleated. Analogous to what was observed in Fig $3 \mathrm{~b}$ and Fig. 4b, dislocations are multiplied continuously while cutting process advances, as shown in Fig. 5b. Frank-type sessile partials and stair-rod partial were also witnessed to nucleate in this case. 


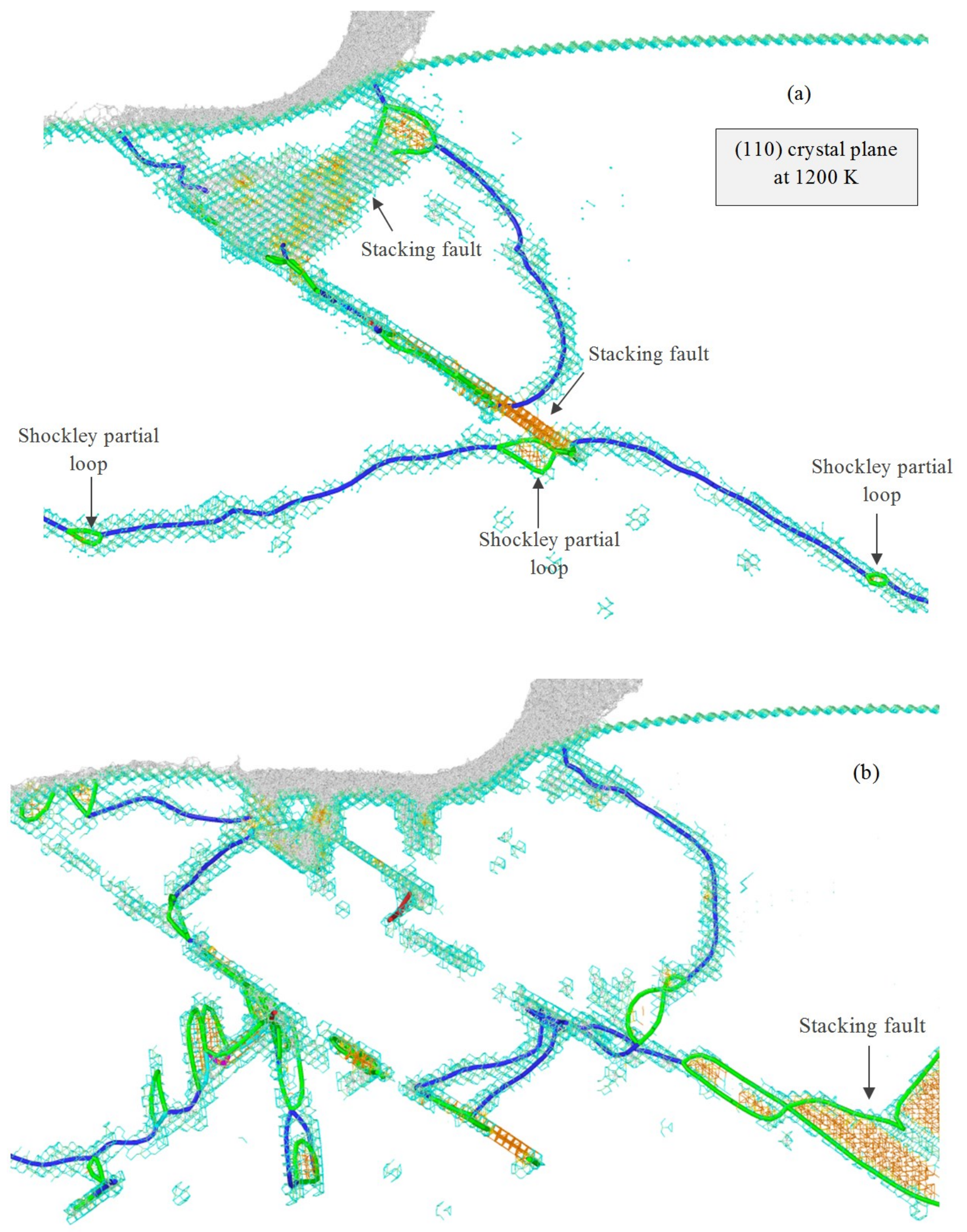

Fig. 5. Formation of crystal defects while cutting the (110) plane at $1200 \mathrm{~K}$ at a cutting distance of a) $10 \mathrm{~nm} \mathrm{b)} 20 \mathrm{~nm}$ 
At $1400 \mathrm{~K}$, perfect dislocation segments, stacking fault bounded between Shockley partial segments, Shockley partial loops between perfect dislocation segments and V-shaped unknown partial are nucleated. A key observation at the cutting distance of $20 \mathrm{~nm}$ is the formation of stacking fault-couple sandwiched among Shockley, stair-rod and other unknown partial segments, as depicted in Fig. 6, which generates a V-shape lock. Nevertheless, the configuration has positioned far behind the cutting tool; thus does not affect the crystal plasticity of $3 \mathrm{C}-\mathrm{SiC}$.

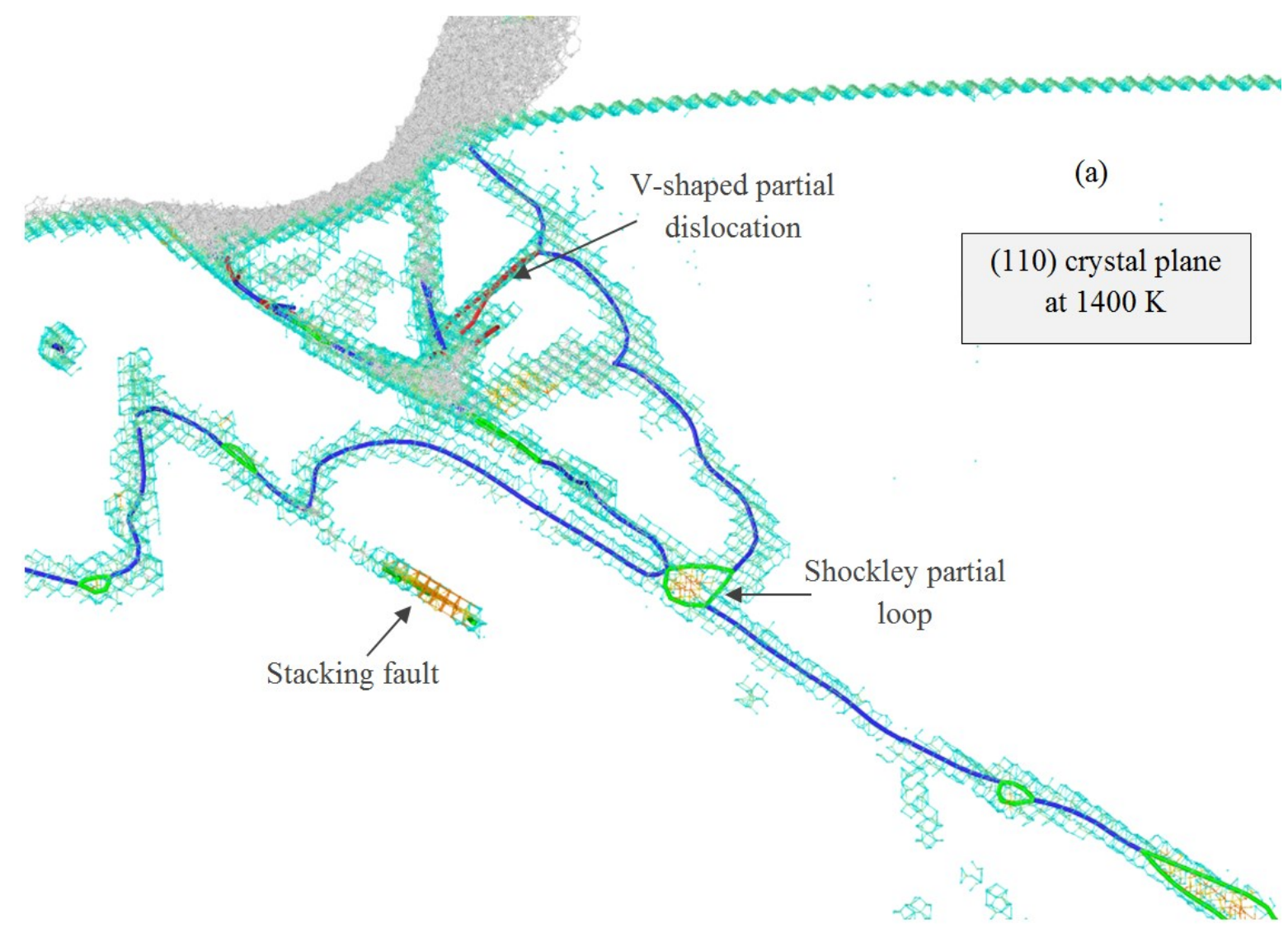




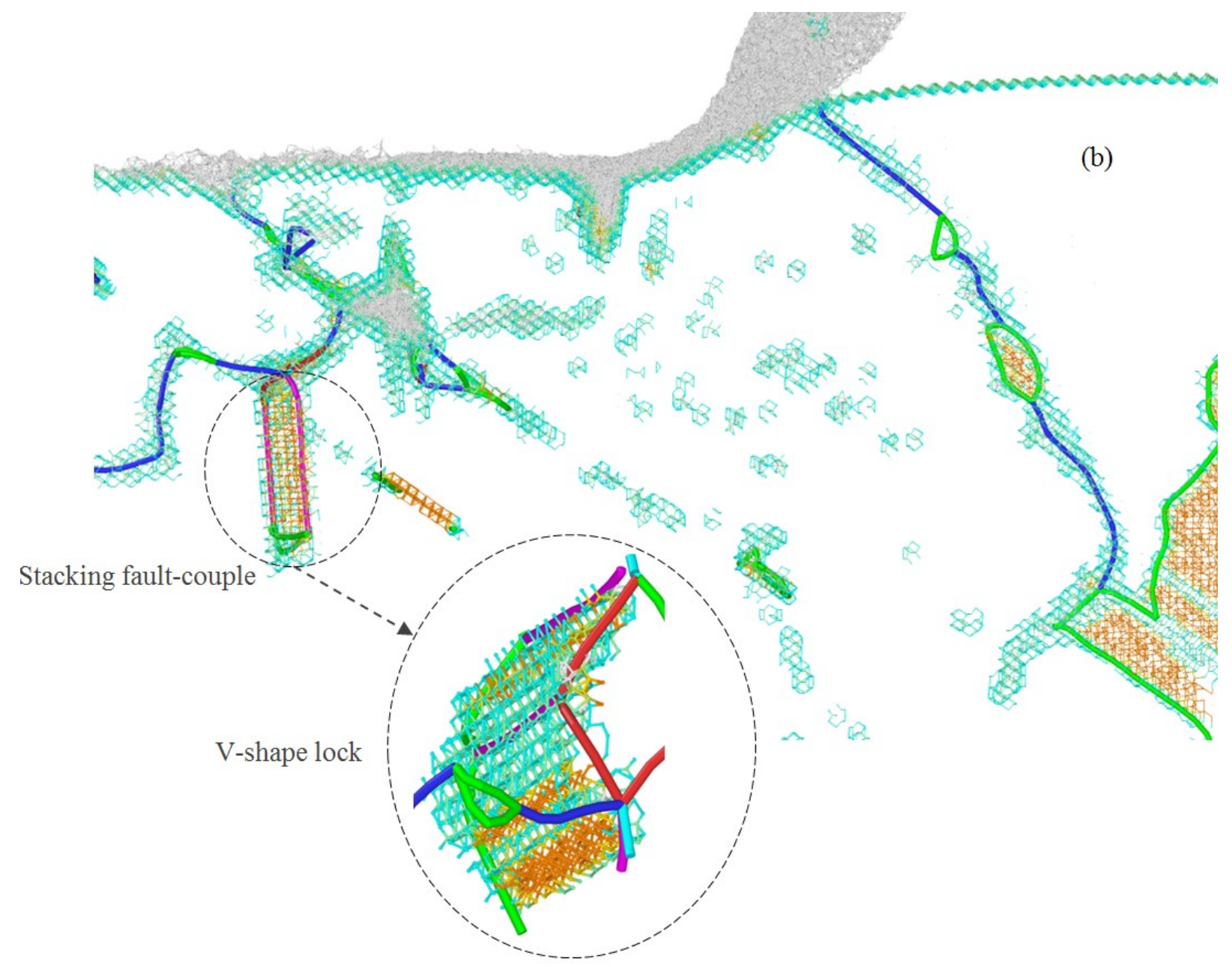

Fig. 6. Formation of crystal defects while cutting the (110) plane at $1400 \mathrm{~K}$ at a cutting distance of a) $10 \mathrm{~nm}$ b) $20 \mathrm{~nm}$. Stacking fault-couple and V-shape lock are formed.

Considering thermally activated defect formation, noticeable dislocation activities including perfect, Shockley, stair-rod, Frank-type sessile and other unknown partials are observed when nanometric cutting of $3 \mathrm{C}-\mathrm{SiC}$ is performed on the $(110)$ plane at $2000 \mathrm{~K}$, as illustrated in Fig. 7. The total length of dislocations was found to be $\sim 6.5$ times higher than that of $300 \mathrm{~K}$. It is of note that at higher temperatures, shorter segments of perfect dislocations and longer segments of partials are nucleated within the substrate, which can be regarded as a transition from perfect dislocation emission to partial nucleation. It is also observed that the size of stacking faults ahead of the tool become larger and the gradual growth of stacking faults on the glide plane is intensified at higher temperatures. The size of stacking fault is indeed influenced by the stable GSFE, i.e. lower the stable GSFE, wider the stacking fault [44]. Thus, since the stable GSFE decreases as the 
temperature increases [42], wider stacking faults are observed at elevated temperatures. It has been reported that the plastic deformation of $4 \mathrm{H}-\mathrm{SiC}$ during compression loading at high temperatures is driven by Shockley partials that bound a ribbon of stacking fault in an experimental study [45]. Since Polytypism of $\mathrm{SiC}$ has been reported to have a trivial effect on the dislocation core energies and Peierls stress [46], the obtained results could be comparable with the aforementioned study.

Stacking fault-couple is also formed, which is bounded between Shockley and unknown partial segments. These dislocations form V-shape junctions, which are typical signature of Lomer-Cottrell (L-C) lock, arising from the interaction of dislocations on two sets of inclined $\{111\}$ planes. Hence, the combination can be recognized as an L-C lock. The bands of stacking faults form a configuration resembling steps on a stairway. These steps are barriers to further slip on the atomic planes involved, as well as in the adjacent planes. These arrangements, i.e. L-C lock and steps, could culminate in the consolidation of material [47], which is analogous to what has been observed for the low stacking fault energy metals [48]. Hence, it can be postulated that such phenomenon are likely to happen for low stacking fault energy materials including metals and semiconductors. Indeed, stacking fault-couple and L-C lock are supposed to be one of the primary mechanisms of strain hardening in FCC metals with low stacking fault energy [49]. Note that with further progress of the cutting tool, the configuration could become unlocked and L-C Lock would be annihilated. Hence, it can be regarded as an unstable combination. In such condition, stacking faults could be decomposed by their counterparts owing to the disconnected slip planes and thus new stacking faults can be formed, as seen in Fig. 7b. As a demonstration of the occurrence of locking/unlocking event, the evolution of cutting forces is plotted in Fig. 8. This figure provides the overall mechanical response of 3C-SiC substrate during nanometric cutting at $2000 \mathrm{~K}$. Abrupt drops in the cutting force can be observed at the highlighted regions, indicating the unlocking process. Upon careful examination, it was observed that the last unlocking was accompanied by the occurrence of cleavage [50]. 
(110) crystal plane at $2000 \mathrm{~K}$

(a)
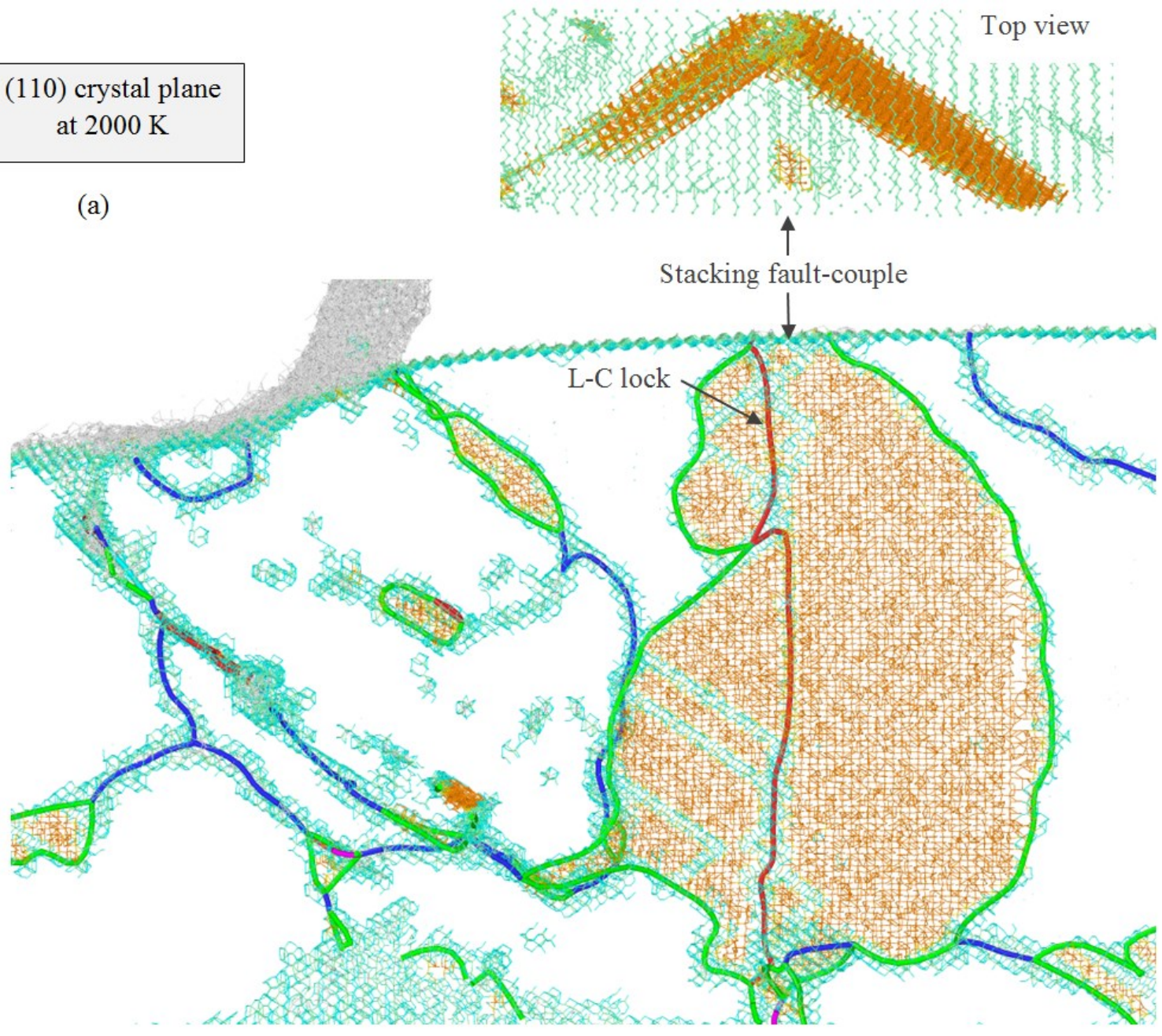
Top view

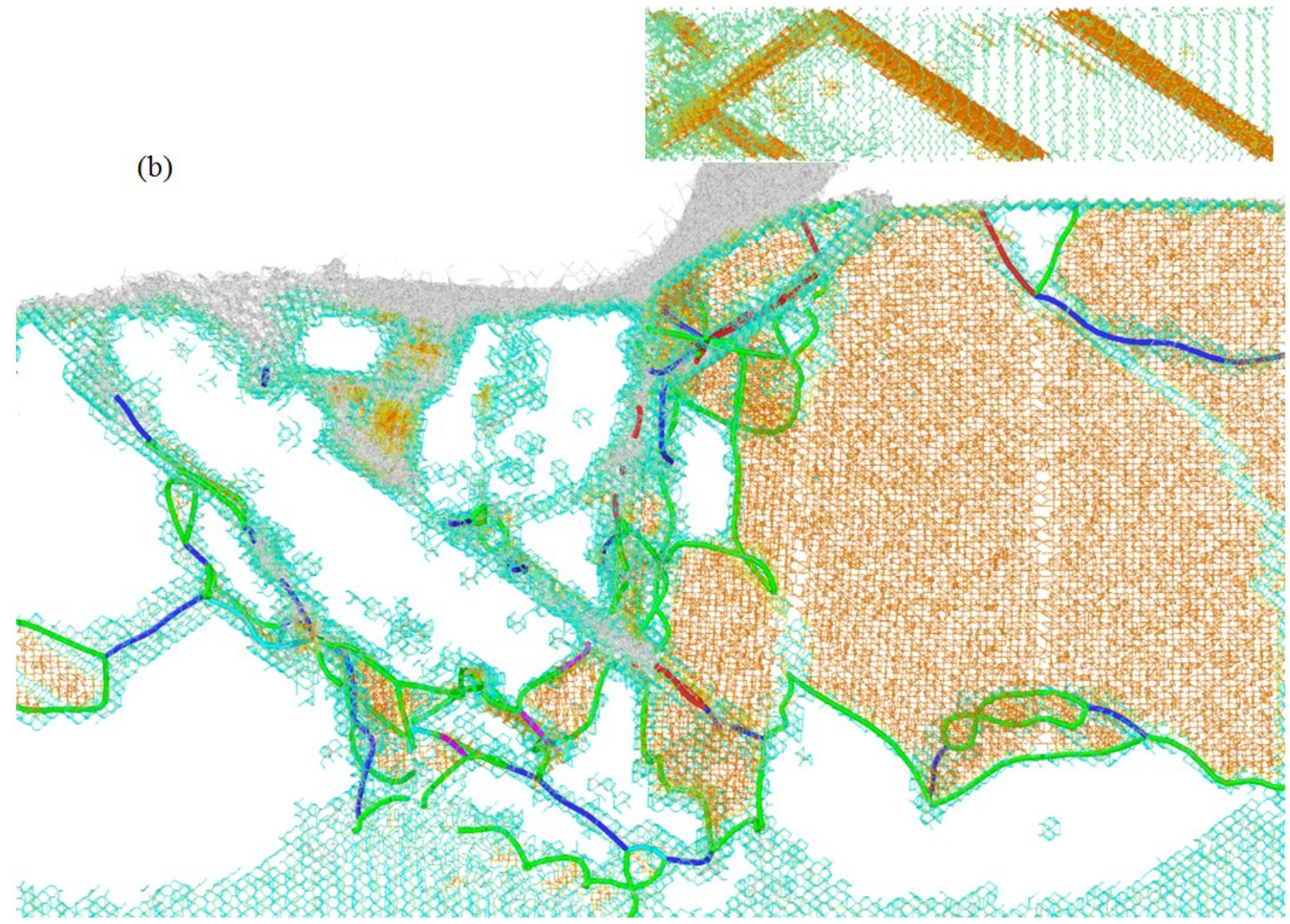

Fig. 7. Formation of crystal defects while cutting the (110) plane at $2000 \mathrm{~K}$ at a cutting distance of a) $10 \mathrm{~nm} \mathrm{b)} 20 \mathrm{~nm}$. Large stacking fault-couple and L-C lock are visible in the figure. 


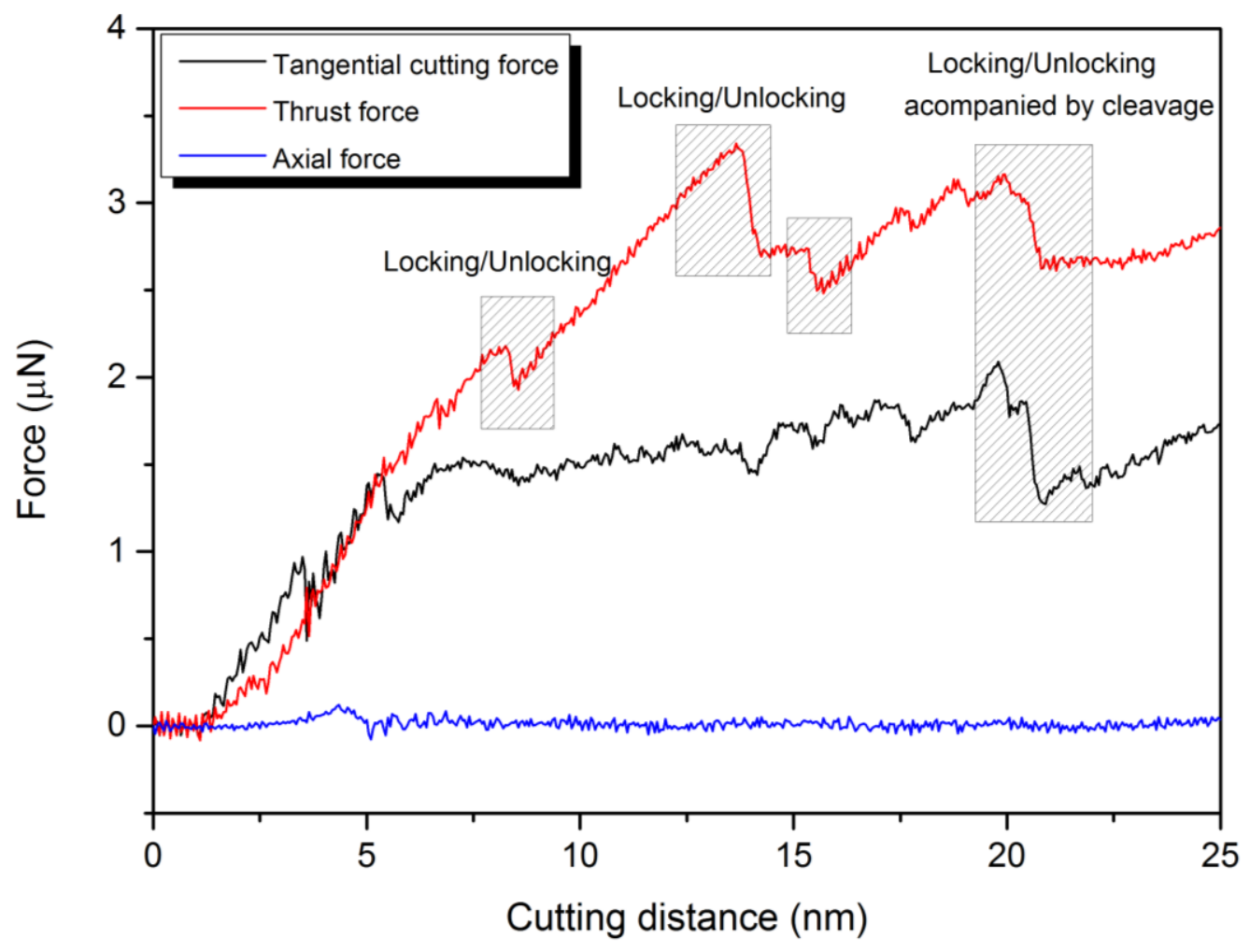

Fig. 8. Evolution of the cutting forces illustrating the locking/unlocking while cutting the (110) plane at $2000 \mathrm{~K}$

At $3000 \mathrm{~K}$, numerous dislocations and large stacking faults are formed. The total length of dislocations nucleated within the $3 \mathrm{C}-\mathrm{SiC}$ substrate was found to be $\sim 10$ times higher than that of $300 \mathrm{~K}$. Intersections of dislocations are also seen, which could cause formation of vacancies, locks and dislocation loops. Successive lock structures, dislocation dendrites, are formed and broken (nucleation-interaction-unzipping cycles) with the progress of the cutting tool; thus, they do not block the plastic flow. Similar to the case of $2000 \mathrm{~K}$, large stacking faults, L-C lock and steps were observed at the cutting distance of $10 \mathrm{~nm}$, as shown in Fig. 9a. It can be seen from Fig. 9b that, at a cutting distance of $20 \mathrm{~nm}$, partial dislocations in particular Shockley partials are nucleated ahead of the cutting tool. However, some perfect dislocations can be also observed. It can be inferred that there is no sharp transition between two regimes of dissociated and non-dissociated dislocations over a large temperature range. This observation is consistent with the ab initio results [51]. It is interesting to see that cross-junctions between pairs of counter stacking faults meditated by the 
gliding of Shockley partials on different slip planes are formed. The intersection of counter pairs of stacking faults could possibly create forest dislocations. This fact can be attributed to the decrease of activation energy of dislocations with the temperature rise. Accordingly, the C-core dislocations turn into mobile leading to the expansion of Si-terminated stacking faults. Intersection of the counter pairs of stacking faults with each other, therefore, could result in the generation of forest dislocations. This phenomenon could impede propagation of successive mobile dislocations. Overall, It can be concluded that while cutting the (110) crystal plane at high temperatures, the plasticity of 3C-SiC is mediated by large stacking faults gliding ahead of the tool tip and continuous movement, multiplication, and entanglement of perfect and dissociated dislocations ahead, underneath and behind of the cutting tool.

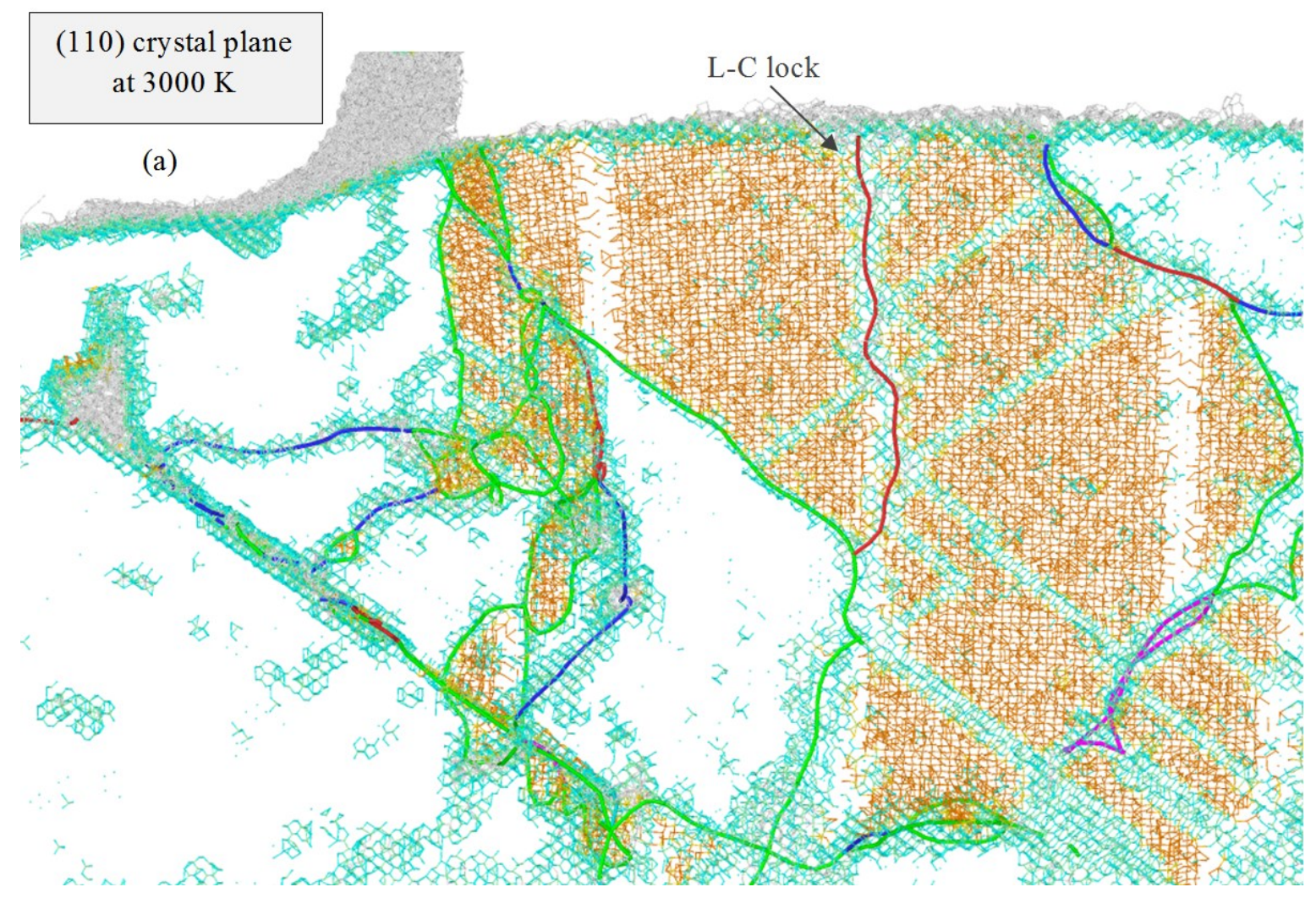




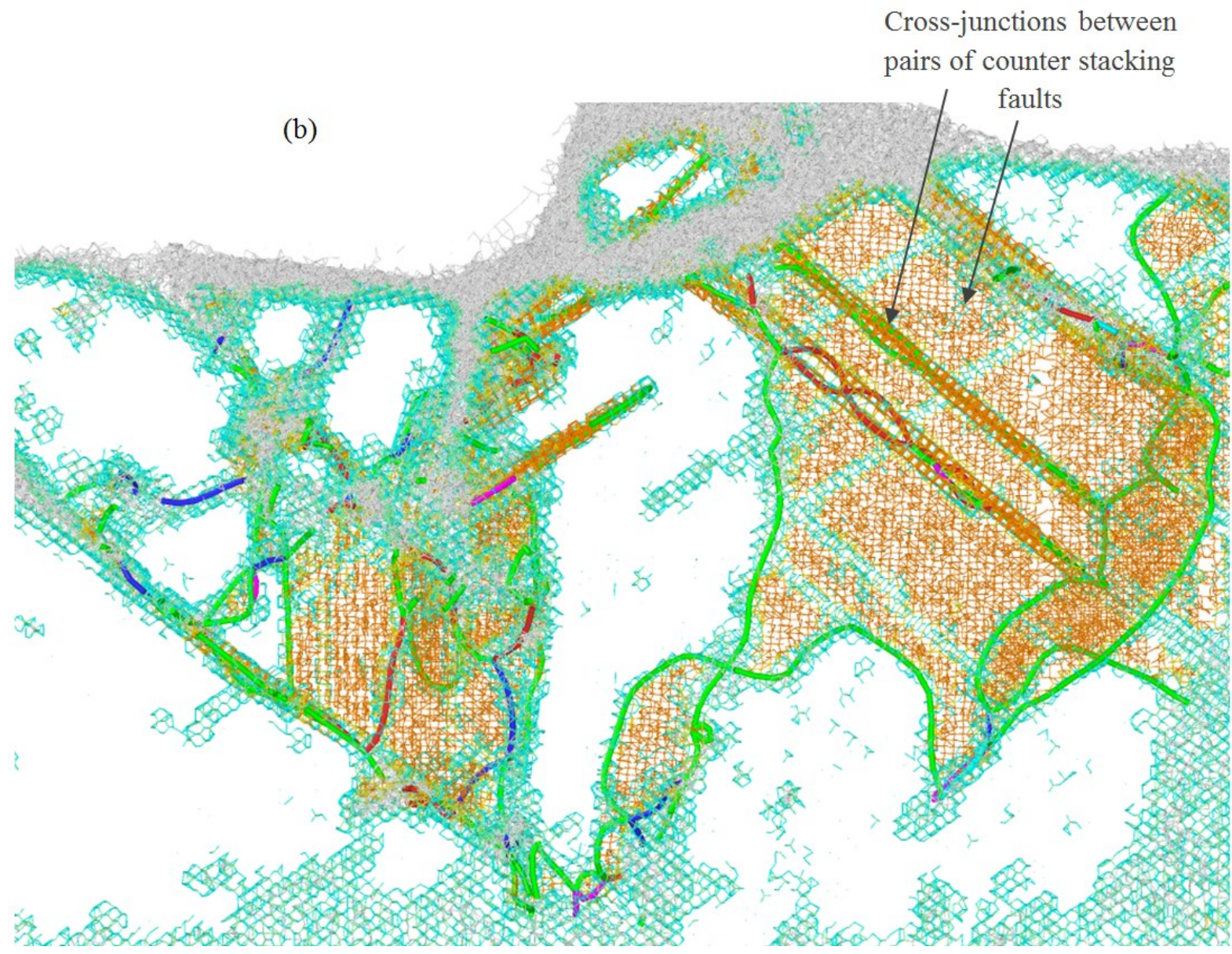

Fig. 9. Formation of crystal defects while cutting the (110) plane at $3000 \mathrm{~K}$ at a cutting distance of a) $10 \mathrm{~nm} \mathrm{b)} 20 \mathrm{~nm}$

In the following session, the plasticity mechanisms of 3C-SiC while cutting the (010) and (111) crystal planes are briefly discussed. In general, lower dislocation activity and stacking fault formation than those of the (110) plane was observed for the aforementioned orientations. As seen in Fig. 10a, while cutting the (010) surface at $300 \mathrm{k}$, two dislocation segments are nucleated beneath the cutting tool at the short cutting distance of $10 \mathrm{~nm}$. Fig. 10b shows that, at the cutting distance of $20 \mathrm{~nm}$, some perfect dislocations are nucleated. However, similar to the aforementioned case, these dislocations are nucleated underneath and behind the cutting tool, which implies that they hardly assist the crystal plasticity of 3C-SiC during nanometric cutting. In general, only dislocations ahead of the cutting tool moving the crystalline region deserve importance in the case of nanometeric cutting. In Fig. 10b, some partial dislocations are visible, which have been attached to the surface. However, they are mere artefacts owing to the limitations of the crystal analysis tool (CAT). 
As shown in Fig. 10c-d, when the substrate temperature is raised to $3000 \mathrm{~K}$, Shockley and stair-rod partials are nucleated accompanied by stacking faults. Furthermore, dissociation of perfect dislocations to partial ones, especially Shockley partials, at higher temperatures can be noticed. It is of note that at high temperatures, dislocations and stacking faults were mostly formed underneath the flank face and tool tip, and glided with the movement of tool. Hence, similar to the case of 300 $\mathrm{K}$, they are less effective on enhancing plasticity than those located ahead of the cutting tool. Another point is that no Frank-type sessile partial dislocations were seen to nucleate for this cutting surface. Overall, it can be deduced that the plasticity of 3C-SiC while cutting the (010) plane at room and high temperatures is marginally governed by the defect formation processes. On the other hand, amorphization and weak bonding between atoms at high temperatures play the key role in plastic flow of $3 \mathrm{C}-\mathrm{SiC}$ when nanometric cutting is performed on the (010) crystal plane. It is interesting to note that the total length of dislocations nucleated within the $3 \mathrm{C}-\mathrm{SiC}$ substrate at 3000 $\mathrm{K}$ in the (010) plane was found to be only $\sim 3$ times higher than that of $300 \mathrm{~K}$.

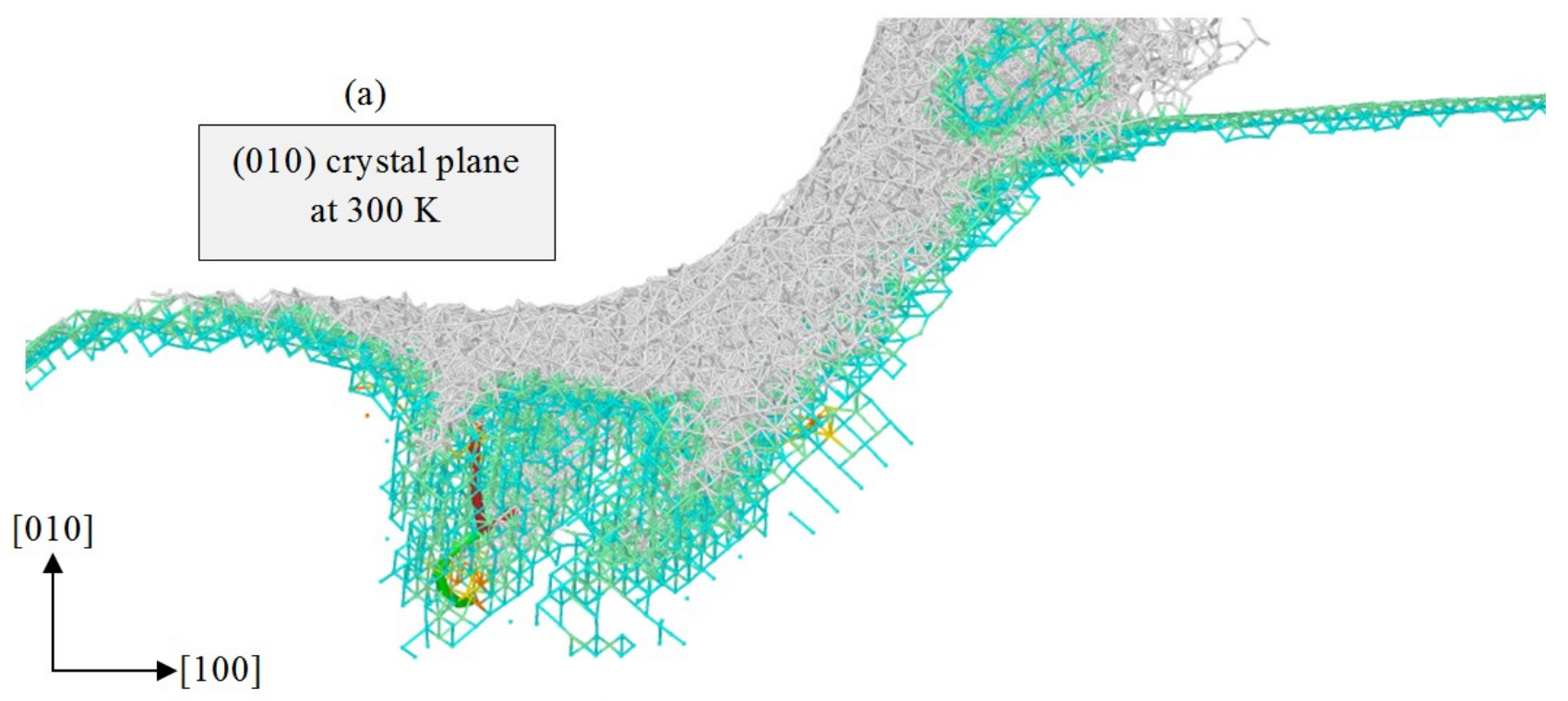


(b)
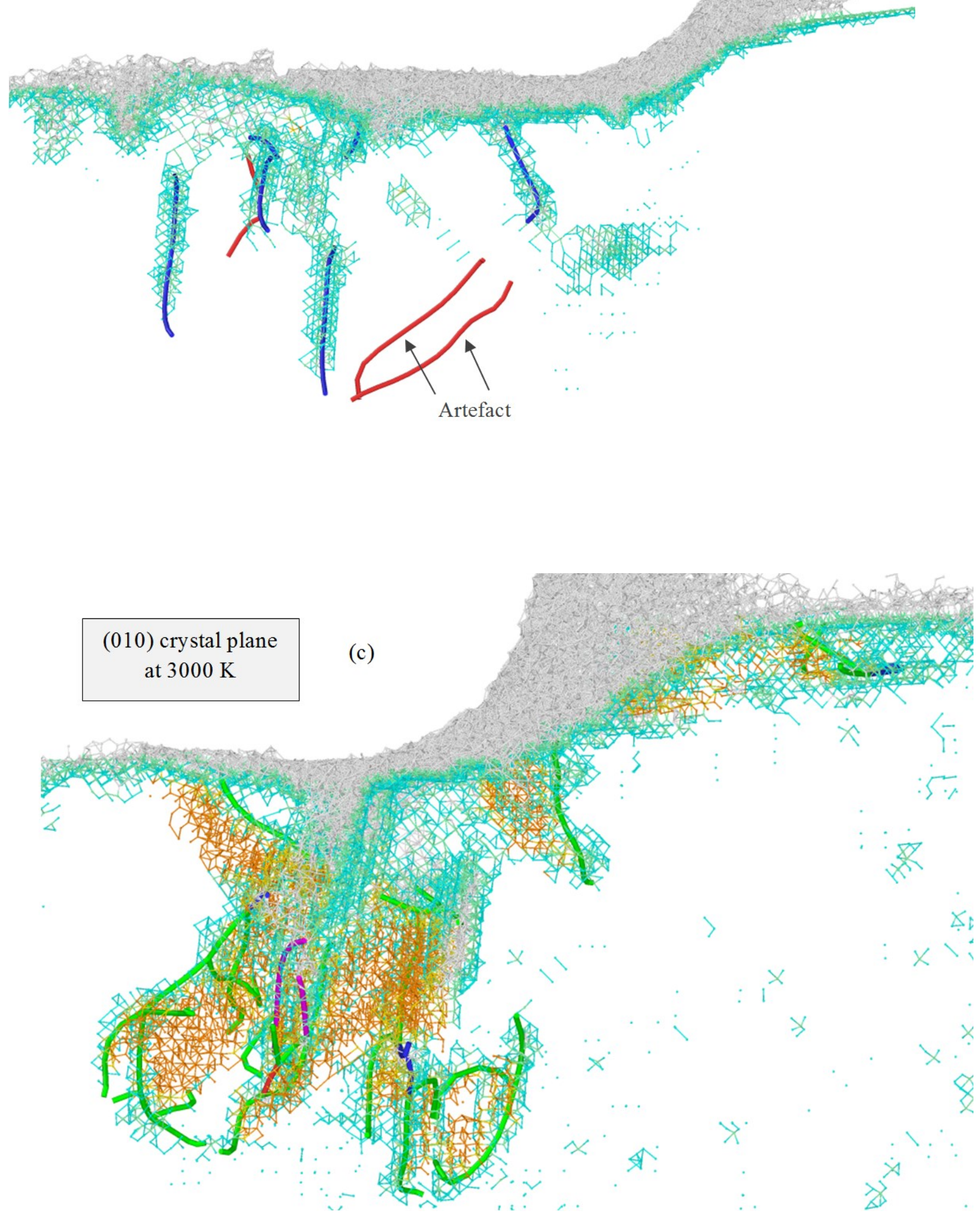


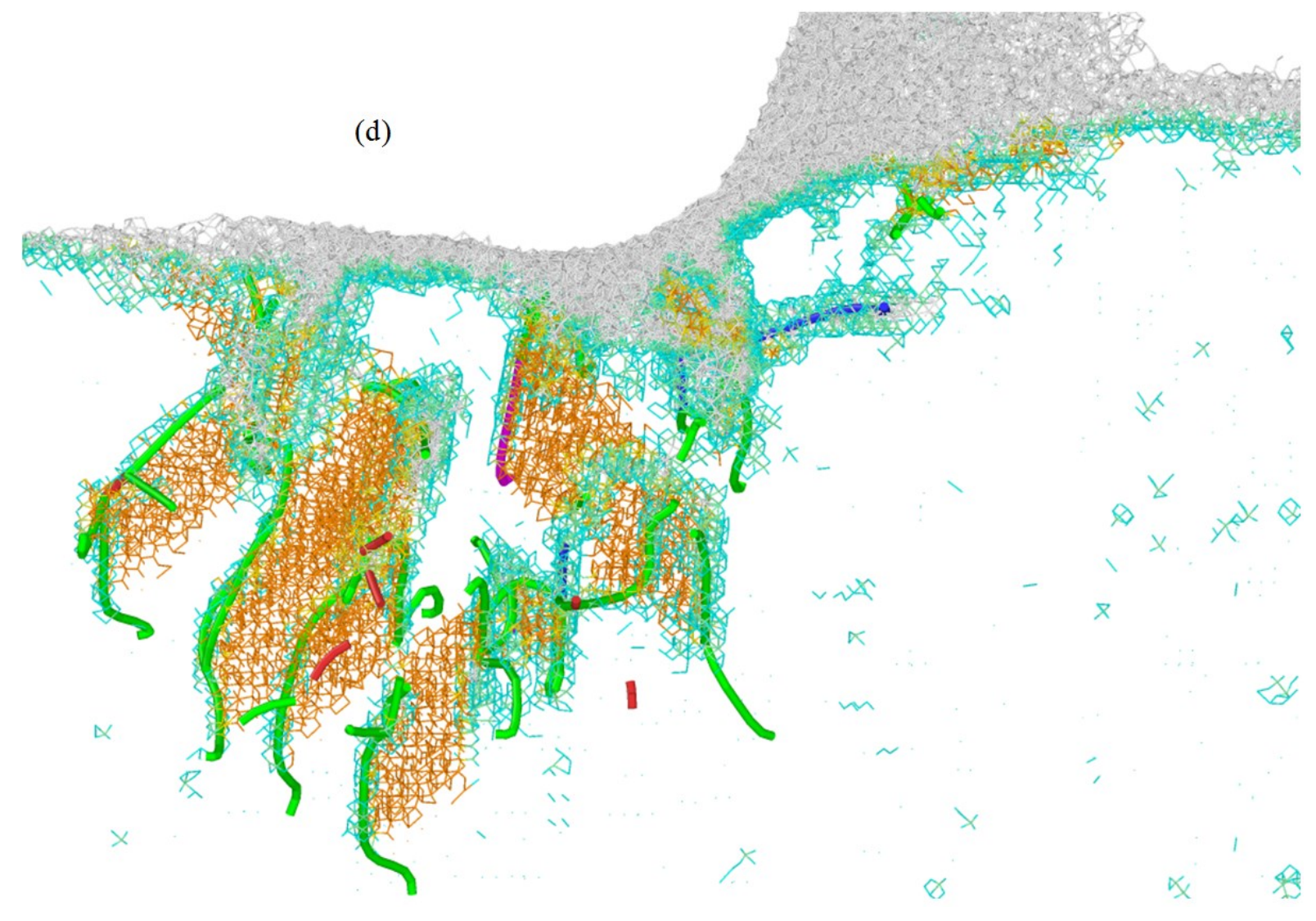

Fig. 10. Formation of crystal defects while cutting the (010) plane at a cutting distance of $10 \mathrm{~nm}$ at a) $300 \mathrm{~K} \mathrm{b)} 3000 \mathrm{~K}$, and at a cutting distance of $20 \mathrm{~nm} \mathrm{c)} 300 \mathrm{~K} \mathrm{~d}$ ) $3000 \mathrm{~K}$

Defect formation processes while cutting $3 \mathrm{C}-\mathrm{SiC}$ on the (111) plane are shown in Fig. 11. In this case, defects are found to be positioned ahead of the tool tip rather than underneath the cutting tool. However, the dislocation activity while cutting the (111) surface is seen to be low at short cutting distances, i.e. $10 \mathrm{~nm}$, as illustrated in Fig. 11a, as opposed to the (110) crystal plane. With the progress of cutting, more dislocations are nucleated ahead of the tool, as shown in Fig. 11b. At higher temperatures i.e. $3000 \mathrm{~K}$, dislocation nucleation and stacking fault formation were observed at short cutting distances. An interesting observation was the formation of horizontal stacking fault at high temperatures of $2000 \mathrm{~K}$ and $3000 \mathrm{~K}$ at the cutting distance of $20 \mathrm{~nm}$, as shown in Fig. $11 \mathrm{~d}$. Also, Frank-type sessile partial dislocation was seen to nucleate as opposed to the (010) plane. The total length of dislocations nucleated within the $3 \mathrm{C}$-SiC substrate at $3000 \mathrm{~K}$ was found to be $\sim 4$ times higher than that of $300 \mathrm{~K}$. 

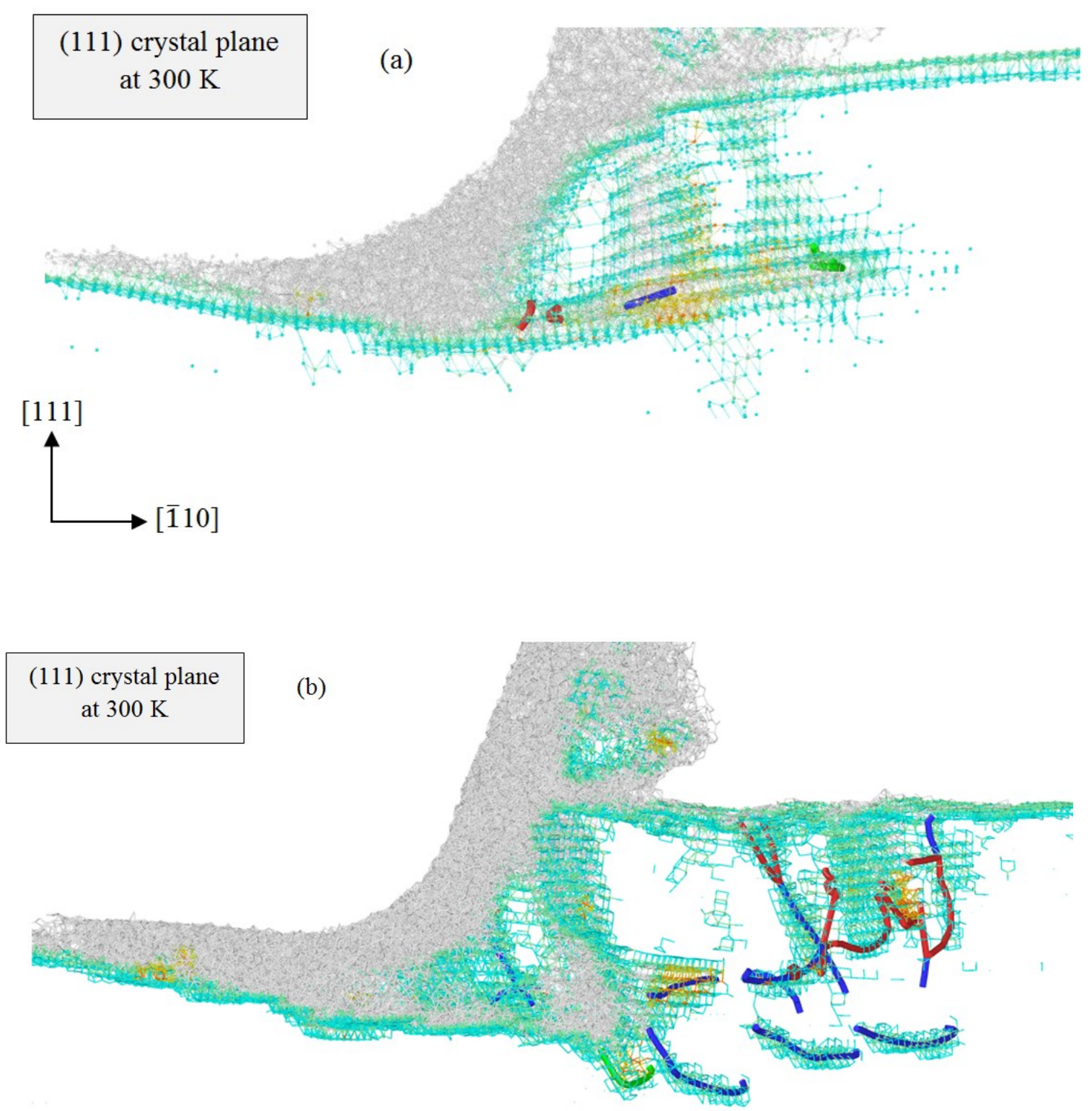
(c)

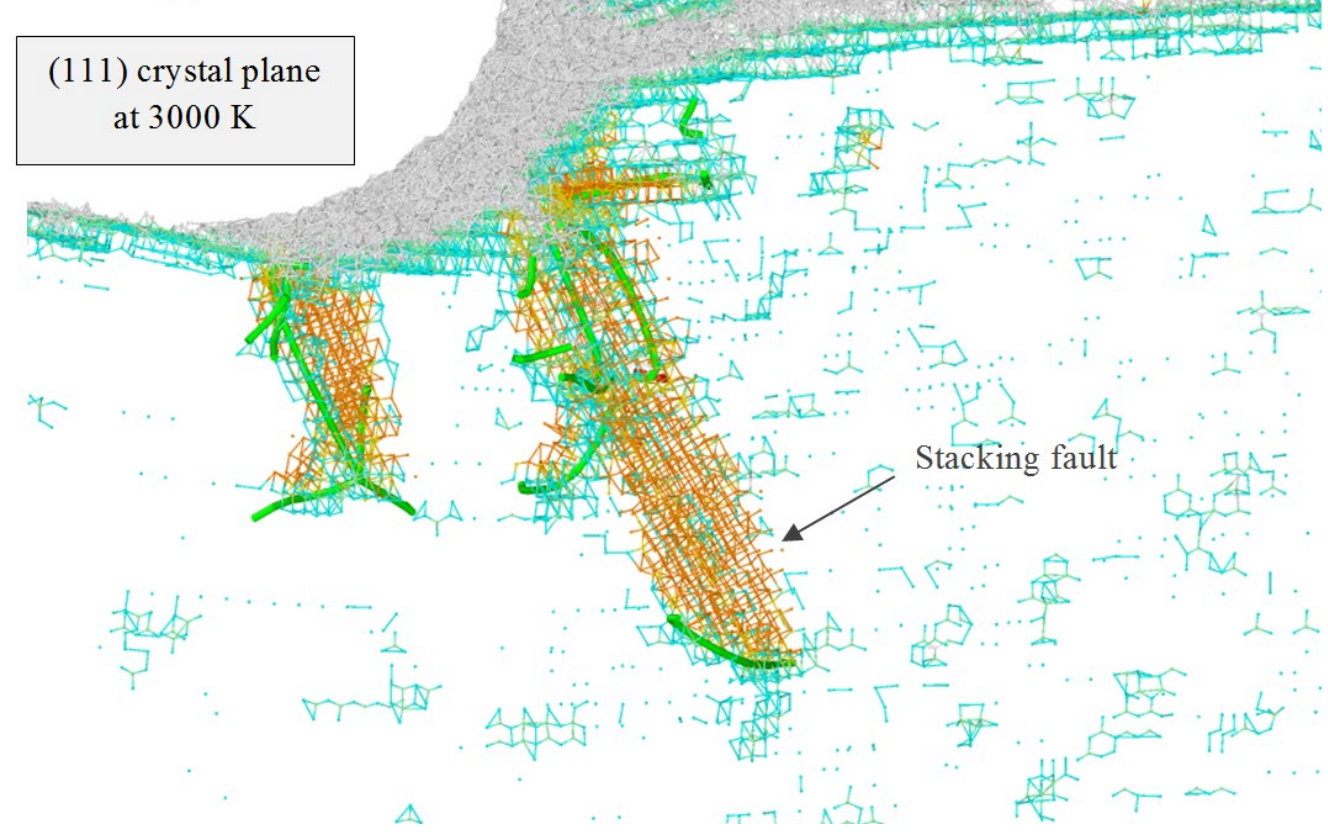

(d)

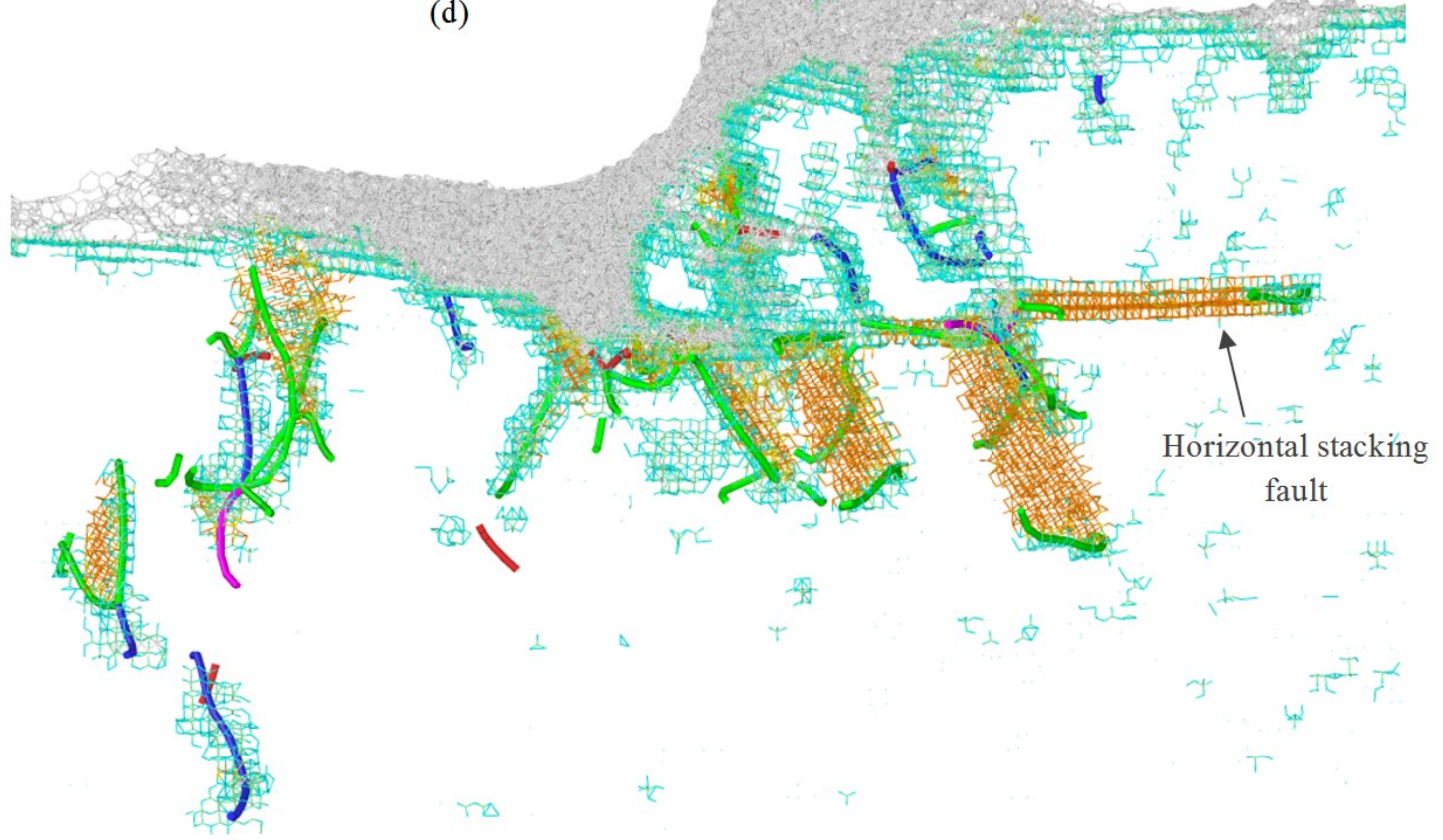

Fig. 11. Formation of crystal defects while cutting the (111) plane at a cutting distance of $10 \mathrm{~nm}$ at a) $300 \mathrm{~K} \mathrm{b)} 3000 \mathrm{~K}$, and at a cutting distance of $20 \mathrm{~nm} \mathrm{c)} 300 \mathrm{~K} \mathrm{~d}) 3000 \mathrm{~K}$

All in all, it can be concluded that a large number of defects is formed while cutting $3 \mathrm{C}$-SiC on the $(110)<00 \overline{1}>$; hence dislocation nucleation and stacking fault formation are the primary deformation response for this crystal setup. However, at room and high temperatures, amorphization also assists 
the plasticity of 3C-SiC. Overall, the initial response of substrate in all the studied cases was the formation of disordered (amorphous) atoms thus it can be generalized that the incipient plasticity of 3C-SiC in nanometric cutting occurs by solid-state amorphization. In addition, at high temperatures, the amplitude of atomic vibration of the substrate atoms increases, which is regarded as an increase in the number of phonons. This phenomenon results in generating atomic displacements. The atomic displacements within the substrate causes an increase in the interatomic distances and a decrease in the restoring forces due to thermal expansion, which lowers the energy required to break the atomic bonds. As a consequence, deformation of 3C-SiC at high temperatures is facilitated. When nanometric cutting is performed on the $(010)<100>$ orientation setup at room and high temperatures, the plasticity of $3 \mathrm{C}-\mathrm{SiC}$ is marginally governed by the defect formation processes. Thus, the plasticity is primarily meditated by amorphization and weak bonding between atoms at high temperatures. With the $(111)<\overline{1} 10>$ setup, room temperature plasticity at short cutting distances is mainly governed by the amorphization whereas at long distances, dislocation nucleation also assists the plastic deformation of 3C-SiC. At higher temperatures, the plasticity of 3C-SiC on the (111) plane is meditated by the defect formation, as well as the amorphization and weak bonding between atoms. It should be also noted that at higher temperatures a small number of stairrod and Frank-type sessile partials are observed to nucleate while cutting 3C-SiC on the different crystal planes.

It is instructive to note that in the cases where the Tersoff potential were applied, no stacking fault formation was observed, attributable to the high stacking fault energy given by this potential, as calculated in Section 3.1. In addition, very low dislocation activity was observed while applying Tersoff potential. For instance, while cutting 3C-SiC on the (010) and (111) planes in the temperature range of $900 \mathrm{~K}$ to $3000 \mathrm{~K}$, no dislocation was nucleated. In the case of (110) orientation, a low density of dislocations was observed at temperatures higher than $900 \mathrm{~K}$. 


\subsection{Structural changes}

To obtain better insights about structural changes of the zinc blende structure, the radial distribution function $g(r)$ before and after nanometric cutting of 3C-SiC at elevated temperatures are plotted. The radial distribution function $(\mathrm{RDF})$, or pair distribution function, is regarded as the major connection between macroscopic thermodynamic characteristics and interatomic activities. It can be seen from Fig. 12 that various peaks occur in the RDF for each individual cutting temperature. The bond length at the peaks has a small discrepancy. The first three peaks of RDF correspond to C-Si, $\mathrm{C}-\mathrm{C}$ and $\mathrm{C}-\mathrm{Si}$ bonds relative to the equilibrium bond lengths of $1.88 \AA, 3.08 \AA$ and $3.62 \AA$, respectively, as shown in Fig. 12. Evidently, the peaks become weaker as temperature of the substrate increases, which is an indication of increased amplitude of atomic vibrations and corresponding atomic displacements from their original equilibrium positions. Apart from the peaks, the bond length increases with the increase of temperature, representing the enhanced structural changes and consequently superior plasticity of $3 \mathrm{C}-\mathrm{SiC}$ before and during the cutting. An interesting observation from Fig 12 is the growth of an embryo peak at the interatomic distance of $1.45 \AA$, which is the equilibrium bond length of $\mathrm{C}-\mathrm{C}$, signifying the formation of $\mathrm{C}-\mathrm{C}$ bond. It should be noted here that the analogous qualitative trend was observed for the three crystal planes. 


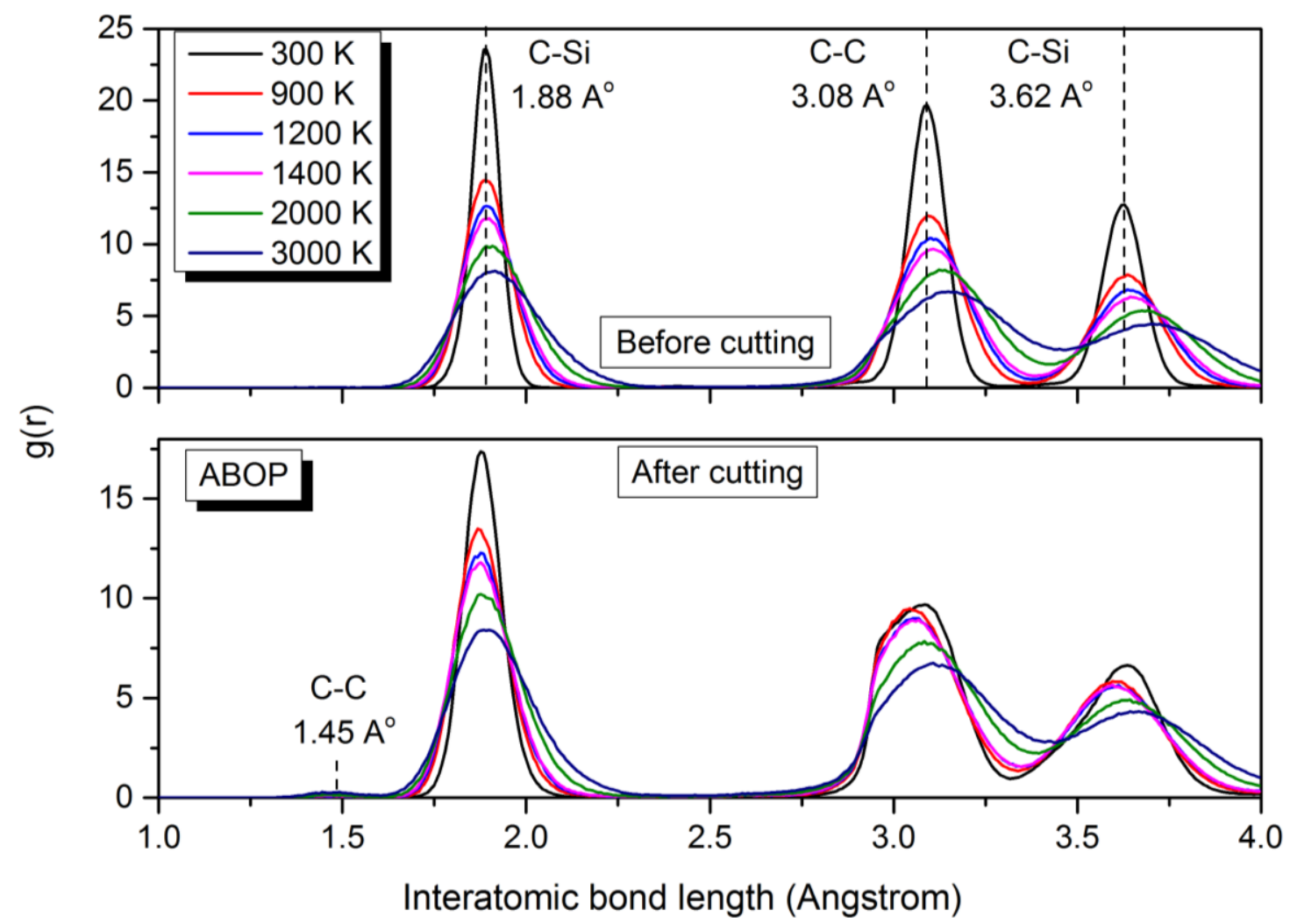

Fig. 12. Radial distribution function showing interatomic bond lengths at different temperatures before and after nanometric cutting on the (010) crystal surface.

As mentioned in Section 3.2, the white atoms in the cutting chip and subsurface of substrate presented in Figs. 3-7 and 9-11 have experienced the transition to a disordered state, a phenomenon known as solid-state amorphization [52]. In order to further characterize the crystal qualities of 3CSiC substrate during nanometric cutting, virtual X-ray diffraction (XRD) [53] with $\mathrm{Cu}$ Ka radiation $(1.5418 \AA)$ was carried on an elemental atomic volume $\left(3 \times 3 \times 3 \mathrm{~nm}^{3}\right.$ including 2552 atoms $)$ in the cutting region. The XRD spectrums obtained from the simulations are illustrated in Fig. 13. Peaks at $2 \theta$ value of $35.96^{\circ}, 40.38^{\circ}, 55.13^{\circ}$ and $76.19^{\circ}$ shown in Fig. 13 a correspond to the crystalline $3 \mathrm{C}$ $\mathrm{SiC}$, which is consistent with the standard XRD pattern of 3C-SiC powders displayed in Fig. 13d [54]. Note that a small amount of mismatch in peak position is commonly acceptable. The prominent peak at $2 \theta=35.96^{\circ}$ is diffracted from $3 \mathrm{C}-\mathrm{SiC}$ (111) according to the database of joint committee on powder diffraction standards (JCPDS). The second, third and fourth ones are diffracted form 3C-SiC (200), (220) and (311), respectively. It can be seen from Fig. 13b that, after cutting, both relative intensities and number of the diffraction peaks alter noticeably, and several 
new diffractions appear. The peaks possess lower relative intensities, particularly the strong peaks shown in Fig. 13a almost disappear, signifying the amorphization of atoms in the cutting deformation zone owing to the large shear stresses and plastic strains. Note that an increase in the intensity of $3 \mathrm{C}-\mathrm{SiC}$ (200) can be seen. However, due to the fact that $\mathrm{Si}$ and $\mathrm{C}$ atoms have ionicity so the local crystal structures are not simple like elements. Hence, it is very challenging to bring a specific reason for this observation.

Fig. 13c shows the XRD profile of 3C-SiC during the cutting, a mixture of crystalline and amorphous atoms in the cutting region, where the pattern could be assumed as a combination of crystalline and amorphous XRD spectra observed in Fig. 13a and 13b. It can be found from Fig. 13c that the intensity maxima of $3 \mathrm{C}-\mathrm{SiC}(111)$ at $2 \theta=35.96^{\circ}$ is approximately 3.8 times lower than that of the crystalline $3 \mathrm{C}-\mathrm{SiC}$, indicating an inferior crystal quality in presence of both crystalline and amorphous structure in the cutting region.

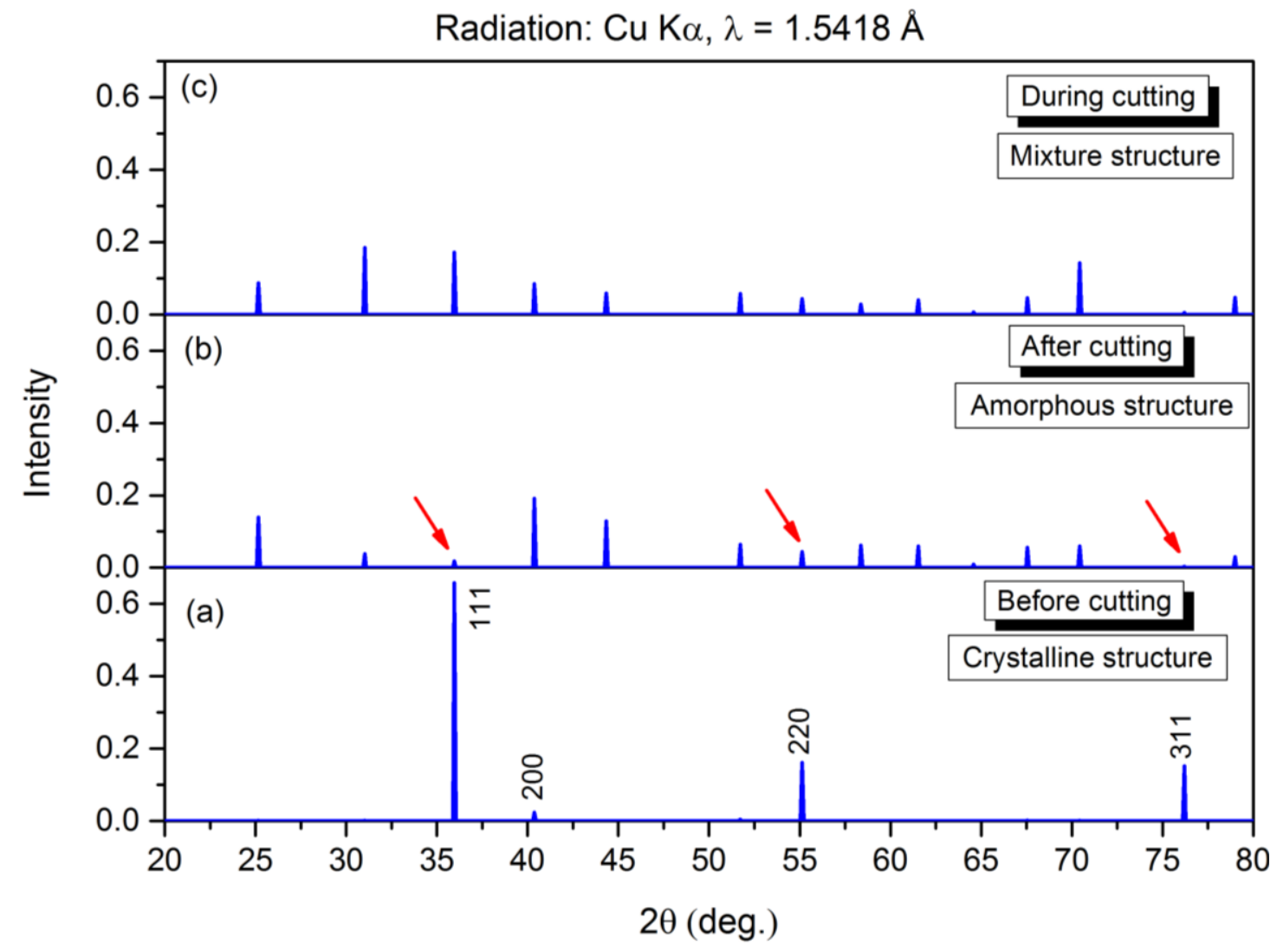




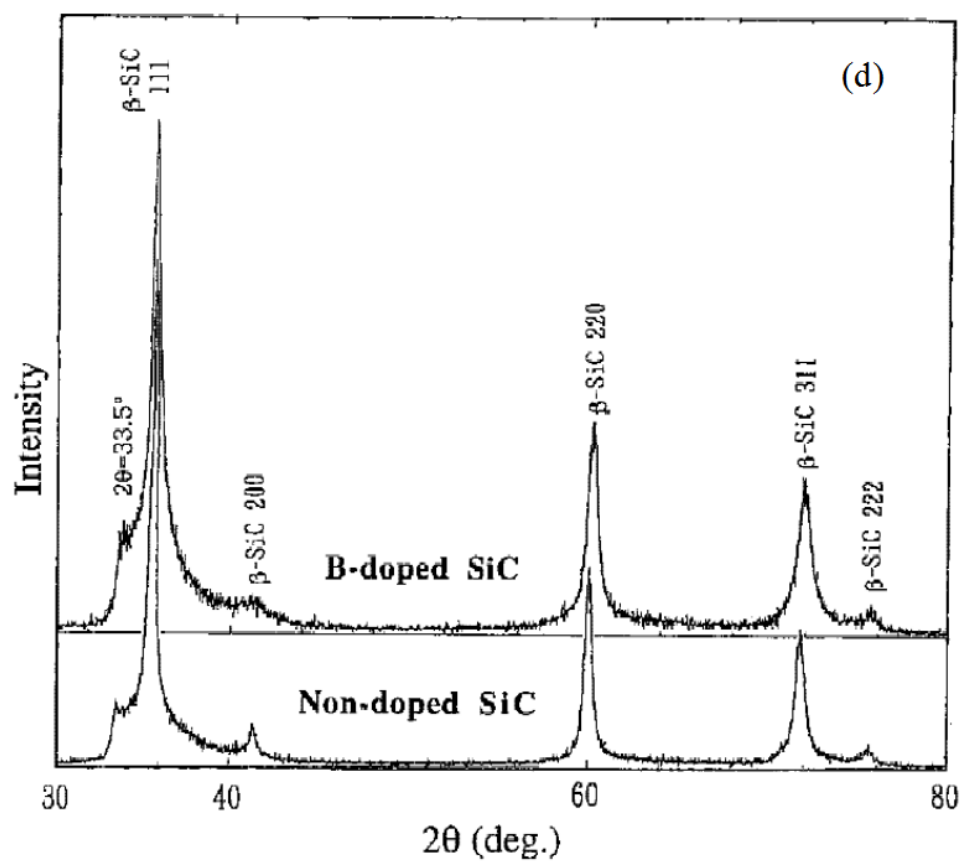

Fig. 13. XRD spectrum of $3 \mathrm{C}-\mathrm{SiC}$ in the cutting region a) before $\mathrm{b}$ ) after and c) during nanometric cutting on the (010) crystal surface. d) Standard XRD pattern of 3C-SiC powders

It should be mentioned here that the available bond order potential (BOP) functions, including $\mathrm{ABOP}$ and Tersoff potentials, are not robust in describing and capturing all the structural phases as they are not able to properly resolve competing mechanisms involving subtle influences of chemical bonding, i.e. ionic bonding. Thus, phase transformation mechanisms cannot be realized using BOP potentials. It is instructive to note that there has been a long debate on the possibility of polymorphic transition to another lattice structure during contact loading of SiC polytypes. Such mechanism has been proposed from experimental studies on 3C-SiC [55]; however, in almost all MD studies no direct evidence has been presented. Instead, indirect observation has been reported, meaning that if $\mathrm{SiC}$ undergoes a local pressure as high as $100 \mathrm{GPa}$ then the transformation to rocksalt structure would take place [56]. Nevertheless, Mishra and Szlufarska [40] have shown that, using Vashishta et al's. potential function [29] which has been reported to be more accurate for describing the high pressure phase transformation of $\mathrm{SiC}$, even under the local atomic pressures as high as $100 \mathrm{GPa}$, the polymorphic transition does not occur in SiC. The only direct observation of phase transformation in MD simulation was provided by Xiao et al. [57], where very small amount of atoms were seen to undergo phase transition from $6 \mathrm{H}-\mathrm{SiC}$ to rocksalt structure. However, more 
evidence is needed to be able to generalize occurrence of the phase transition at a strictly nanometre level in MD trials. Hence, polymorphic transition is still a hypothesis for the deformation mechanisms of $\mathrm{SiC}$ in $\mathrm{MD}$ simulation, in contrast to silicon where phase transformation has been witnessed [58]. However, defect-free specimen employed in MD simulation might play a role in hindering the occurrence of such transition in MD simulations of SiC.

\subsection{Yielding stresses}

Material dependent yielding criteria such as von Mises stress, Principal stress and Tresca stress measures were employed to predict yielding of $3 \mathrm{C}-\mathrm{SiC}$ under various circumstances in this study. In order to compute the atomic stress tensor ${ }^{1}$, an elemental atomic volume $\left(1 \times 1.5 \times 3 \mathrm{~nm}^{3}\right.$ including 442 atoms) was considered in the cutting region and accordingly the physical stress tensor was obtained. Equations shown in Appendix A were adopted to calculate the aforementioned yielding measures. Table 1B in Appendix B summarizes the magnitude of all the stresses obtained from the simulation data in all the test cases at varying temperatures. Fig. 14 compares the critical von Mises stresses obtained from the ABOP potential function at different cutting temperatures and crystal orientations while cutting $3 \mathrm{C}-\mathrm{SiC}$. The von Mises stress is a very commonly used yield criterion to estimate the yielding of a material and is based on the assumption that the maximum deviatoric strain energy causes yielding in the material. It is apparent from Fig. 14 that the critical von Mises stress triggering flow during nanometric cutting of 3C-SiC decreases with the increase of substrate temperature. It is interesting to note that the largest values of the required von Mises stress to cause yielding appears on the (110) crystal plane whereas the smallest values are observed on the (111) surface. Furthermore, at temperatures higher than $1400 \mathrm{~K}$, the critical von Mises stress on the (010) and (110) shows almost the same magnitudes. It is noteworthy that the maximum reduction in yielding von Mises stress at $3000 \mathrm{~K}$ with respect to the room temperature cutting takes place on the (110) plane, which is up to $49 \%$, followed by the (010) and (111) crystal planes, $40 \%$ and $37 \%$,

\footnotetext{
${ }^{1}$ http://lammps.sandia.gov/doc/compute_stress_atom.html
} 
respectively.

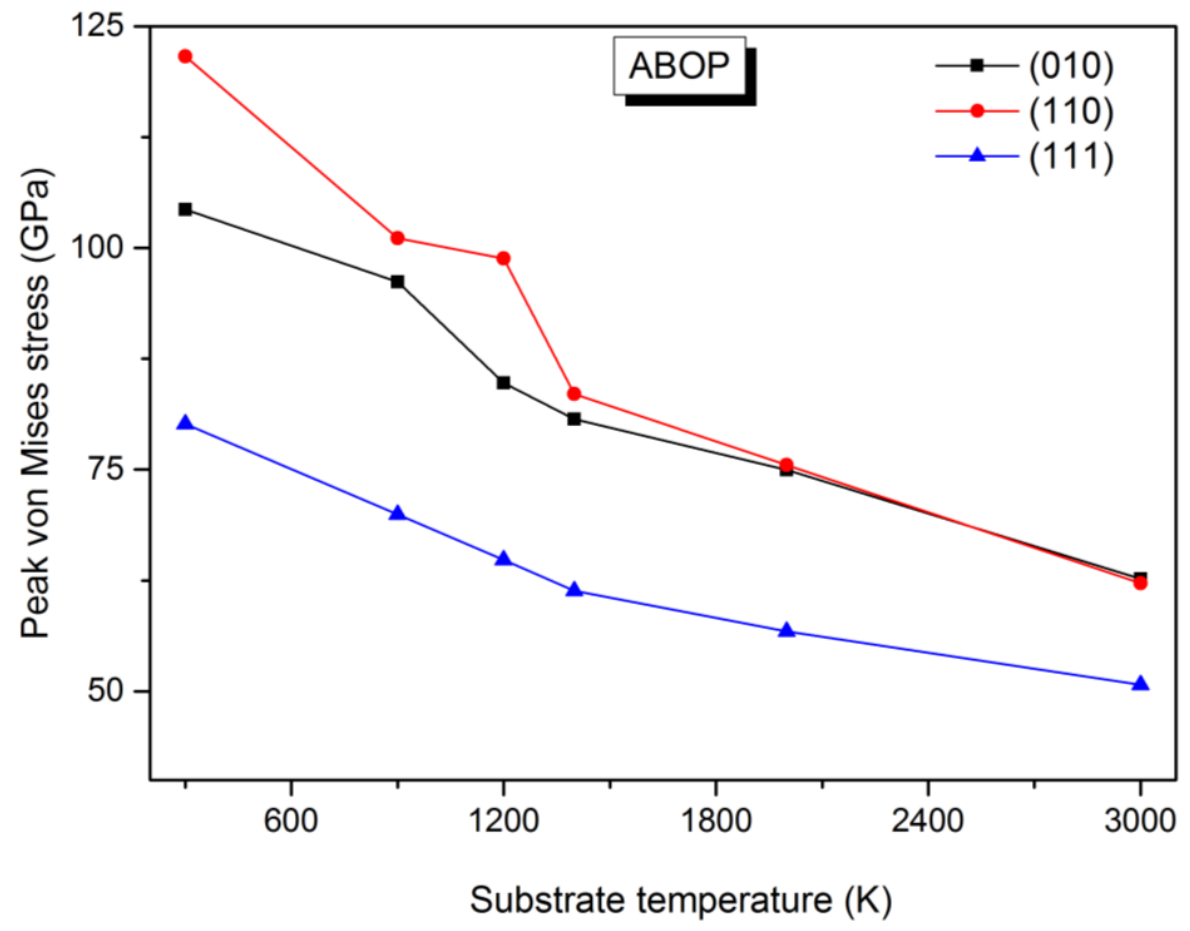

Fig. 14. Variation of critical von Mises stress in the cutting region while cutting $3 \mathrm{C}-\mathrm{SiC}$ on different crystal planes and at different temperatures obtained by ABOP potential function

Nanometric cutting is recognized as a shear dominated process which generates heat in the cutting region owing to breaking and reformation of bonds, leading to an increase of the temperature locally in the cutting region. In order to appreciate the effect of the stress and temperature on the behaviour of the substrate, evolution of average local temperature and von Mises stress in the cutting region is plotted in Fig. 15. Almost similar trend was observed for the other crystal planes and cutting temperatures, and hence not repeated. It can be deduced from Fig. 15 that the peak temperature and peak stress required to cause yielding in the cutting region do not take place simultaneously. This difference exists because the maximum temperature signifies complete fracture of the bonds which may not be the necessary condition for the material to flow (peak temperature at peak stress). Table 1B in Appendix B provides a complete list of the peak temperatures and temperatures at the onset of yielding in the cutting zone for all the simulated cases. 


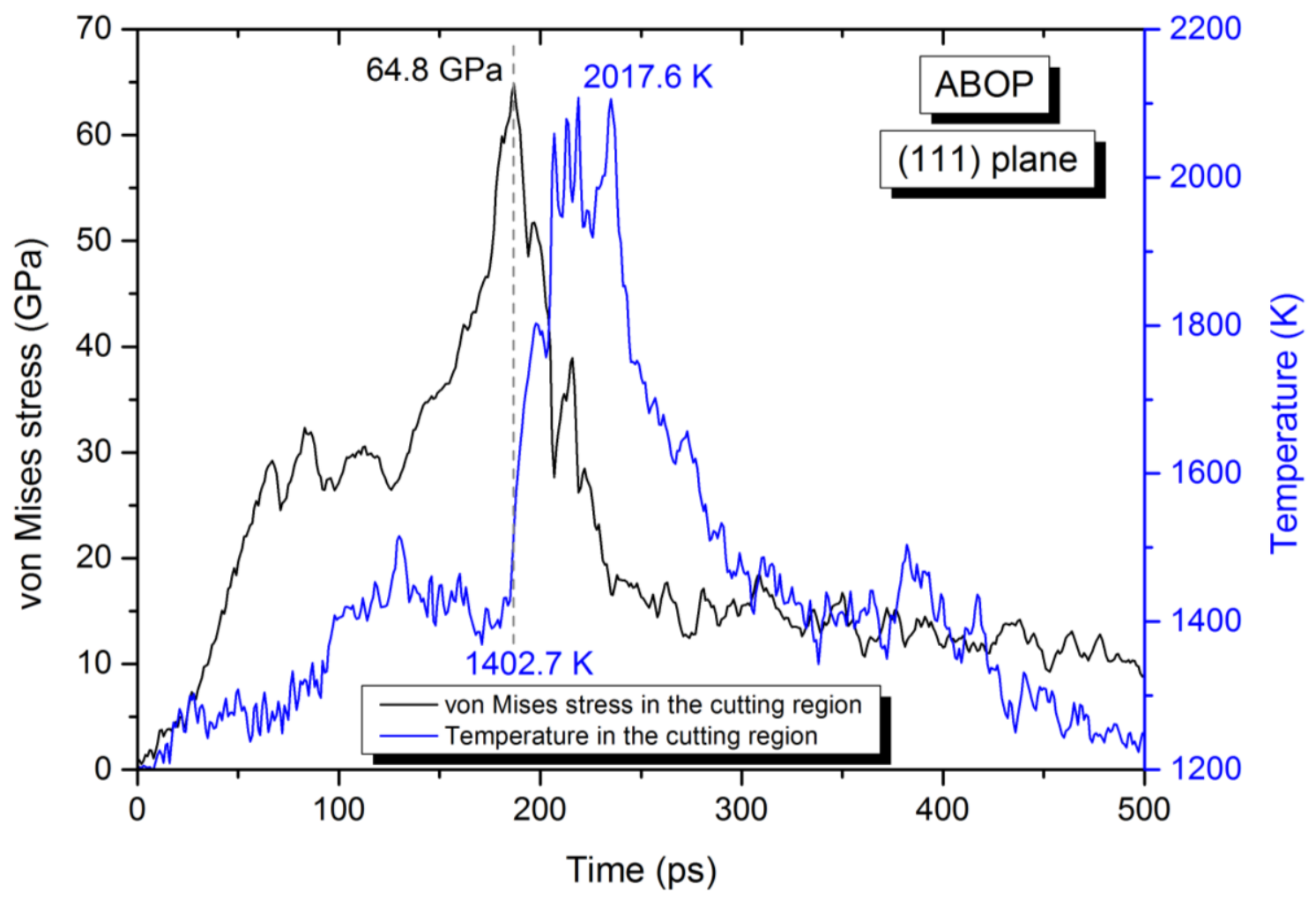

Fig. 15. Evolution of the temperature and von Mises stress in the cutting region recorded on the same plot while cutting $3 \mathrm{C}-\mathrm{SiC}$ on the (111) crystal plane at $1200 \mathrm{~K}$

\section{Concluding remarks}

This work made use of the MD simulation to deal with the nanoscale plasticity of single crystal 3C-

$\mathrm{SiC}$ during nanometric cutting on different crystallographic orientations at a range of temperature (from $300 \mathrm{~K}$ to $3000 \mathrm{~K}$ ) using two potential energy functions, i.e. ABOP and Tersoff. Complimentary calculations of mechanical properties, GSFE surfaces and ideal shear stresses lend further credence to the reported findings. Based on the aforementioned results, conclusions can be drawn as:

1. Successive formation and destruction of stacking fault-couple bounding between partial dislocation segments and Lomer-Cottrell (L-C) lock were observed while cutting 3C-SiC on the $(110)<00 \overline{1}>$ orientation setup at high temperatures. Furthermore, cross-junctions 
between pairs of counter stacking faults meditated by the gliding of Shockley partials on different slip planes were formed at $3000 \mathrm{~K}$ for the aforementioned crystal setup.

2. Dislocation multi-junction, Frank-type sessile and stair-rod partials were observed to nucleate at substrate temperatures $T \geq 900 \mathrm{~K}$ when cutting was performed on the (110) surface.

3. Dislocation nucleation and stacking fault formation were observed to be dominant during cutting the $(110)<00 \overline{1}>$ crystal setup. However, low defect activity was seen for the $(010)<100>$ and $(111)<\overline{1} 10>$ crystal setups. More importantly, the incipient plasticity of $3 \mathrm{C}$ $\mathrm{SiC}$ in nanometric cutting was observed to occur by solid-state amorphization.

4. Substantial difference was observed in both the relative intensities and number of the diffraction peaks of the virtual XRD spectrums before and after cutting, signifying the amorphization of atoms in the cutting deformation region. In addition, the peaks in RDF became weaker and bond length was seen to increase as substrate temperature increased or after cutting.

5. The maximum reduction in yielding stresses with respect to the increase of cutting temperature occurred on the (110) crystal plane.

\section{Acknowledgment}

The authors gratefully acknowledge the financial support from the EPSRC (EP/K018345/1) and Royal Society-NSFC international exchange programme (IE141422) for this study. The authors also acknowledge the use of the EPSRC (EP/K000586/1) funded ARCHIE-WeSt High Performance Computer at the University of Strathclyde. The first author (SZC) also acknowledges the help of Dr. Saurav Goel from Queen's University, Belfast, UK and Dr. Alexander Stukowski from TU Darmstadt, Germany for their useful advice. 


\section{Appendix A}

Stress tensor $=\left[\begin{array}{lll}\sigma_{x x} & \tau_{x y} & \tau_{x z} \\ \tau_{x y} & \sigma_{y y} & \tau_{y z} \\ \tau_{x z} & \tau_{x z} & \sigma_{z z}\end{array}\right]$

$I_{1}=\sigma_{x x}+\sigma_{y y}+\sigma_{z z}$

$I_{2}=\sigma_{x x} \sigma_{y y}+\sigma_{y y} \sigma_{z z}+\sigma_{z z} \sigma_{x x}-\tau_{x y}^{2}-\tau_{x z}^{2}-\tau_{y z}^{2}$

$I_{3}=\sigma_{x x} \sigma_{y y} \sigma_{z z}+2\left(\tau_{x y} \tau_{y z} \tau_{x z}\right)-\tau_{x z}^{2} \sigma_{y y}-\tau_{y z}^{2} \sigma_{x x}-\tau_{x y}^{2} \sigma_{z z}$

$A_{1}=-I_{1} ; A_{2}=I_{2} ; A_{3}=-I_{3}$

$Q=\frac{3 A_{2}-A_{1}^{2}}{9}$

$R=\frac{9 A_{1} A_{2}-27 A_{3}-2 A_{1}^{3}}{54}$

$D=Q^{3}+R^{2}$

If $\mathrm{D}<0$ then as follows: else the condition is $2 \mathrm{D}$ stress

$\theta=\cos ^{-1}\left(\frac{R}{\sqrt{-Q^{3}}}\right)$

$R_{1}=2 \sqrt{-Q} \times \cos \left(\frac{\theta}{3}\right)-\frac{A_{1}}{3}$

$R_{2}=2 \sqrt{-Q} \times \cos \left(\frac{\theta+4 \pi}{3}\right)-\frac{A_{1}}{3}$

$R_{3}=2 \sqrt{-Q} \times \cos \left(\frac{\theta+2 \pi}{3}\right)-\frac{A_{1}}{3}$

$\sigma_{1}=\max \left(R_{1}, R_{2}, R_{3}\right) ; \sigma_{3}=\min \left(R_{1}, R_{2}, R_{3}\right)$

$\sigma_{\text {tresca }}=\frac{\sigma_{1}-\sigma_{3}}{2}$

$\sigma_{\text {von Mises }}=\sqrt{\frac{\left(\sigma_{\mathrm{xx}}-\sigma_{\mathrm{yy}}\right)^{2}+\left(\sigma_{\mathrm{yy}}-\sigma_{\mathrm{zz}}\right)^{2}+\left(\sigma_{\mathrm{zz}}-\sigma_{\mathrm{xx}}\right)^{2}+6\left(\tau_{\mathrm{xy}}^{2}+\tau_{\mathrm{yz}}^{2}+\tau_{\mathrm{zx}}^{2}\right)}{2}}$

$\sigma_{\text {oct }}=\frac{\sqrt{\left(\sigma_{\mathrm{xx}}-\sigma_{\mathrm{yy}}\right)^{2}+\left(\sigma_{\mathrm{yy}}-\sigma_{\mathrm{zz}}\right)^{2}+\left(\sigma_{\mathrm{zz}}-\sigma_{\mathrm{xx}}\right)^{2}+6\left(\tau_{\mathrm{xy}}^{2}+\tau_{\mathrm{yz}}^{2}+\tau_{\mathrm{zx}}^{2}\right)}}{3}=\frac{\sqrt{2}}{3} \sigma_{\text {von Mises }}$ 


\section{Appendix B}

Table 1B. Stresses and temperatures in the cutting region while cutting $3 \mathrm{C}-\mathrm{SiC}$ on different crystallographic orientations obtained by ABOP potential function

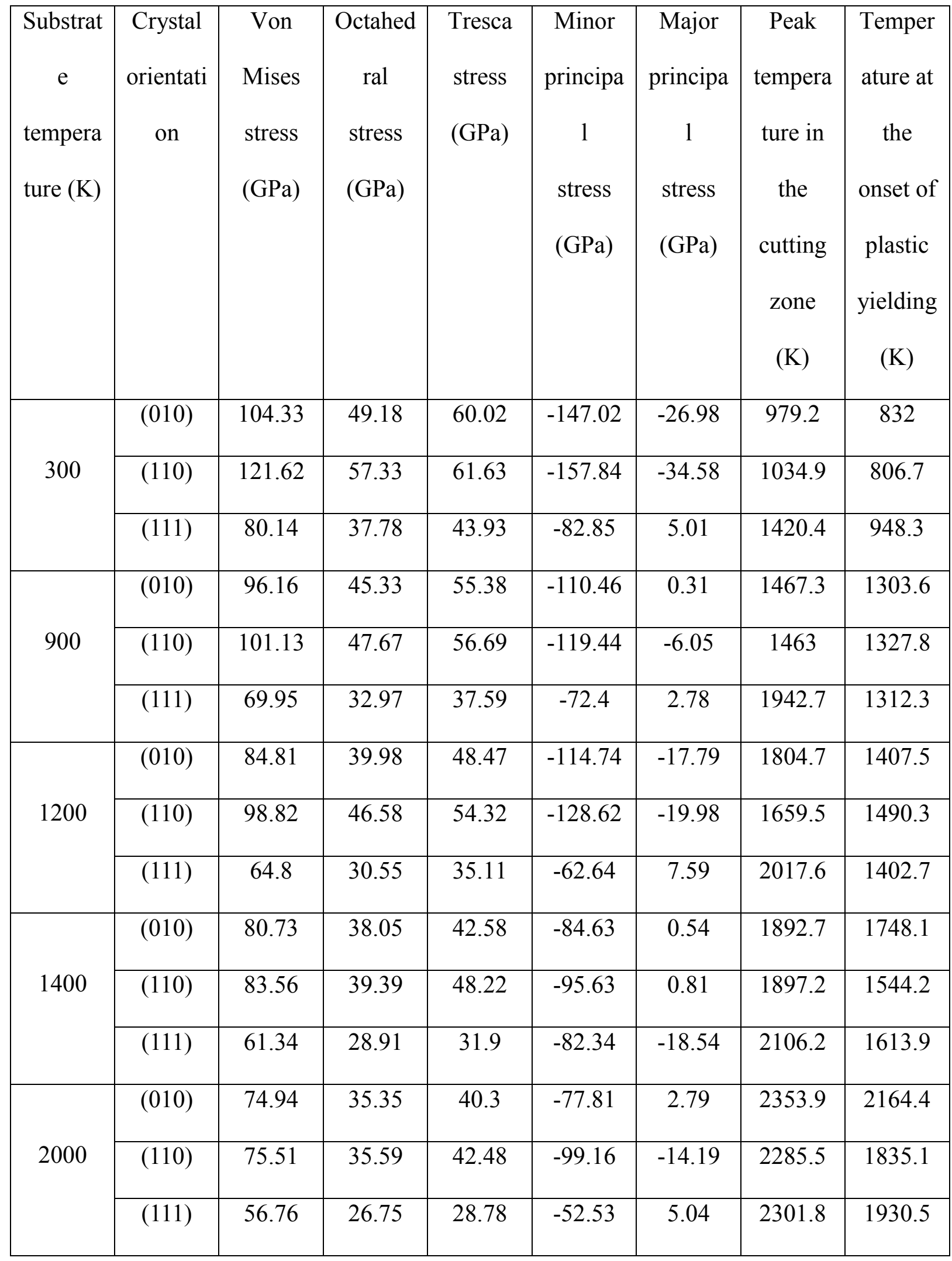




\begin{tabular}{|c|c|c|c|c|c|c|c|c|}
\hline \multirow{3}{*}{3000} & $(010)$ & 62.68 & 29.55 & 36.02 & -66.76 & 5.28 & 3395.6 & 2590.1 \\
\cline { 2 - 9 } & $(110)$ & 62.17 & 29.31 & 34.87 & -80.34 & -10.59 & 3267.5 & 2527.6 \\
\cline { 2 - 9 } & $(111)$ & 50.71 & 23.9 & 26.36 & -49.88 & 2.85 & 3230 & 2565.3 \\
\hline
\end{tabular}

\section{References}

[1] S. Goel, The current understanding on the diamond machining of silicon carbide. J. Phys. D: Appl. Phys. 47 (2014) 243001

[2] M. Yoshida et al, Pressure-induced phase transition in SiC. Phys. Rev. B 48 (1993) 10587-90

[3] F. Shimojo et al, Molecular dynamics simulation of structural transformation in silicon carbide under pressure. Phys. Rev. Lett. 84 (2000) 3338

[4] M. Tang, Elastic instabilities and structural responses of Beta-SiC under stress $\mathrm{PhD}$ Thesis Massachusetts Institute of Technology, Cambridge, MA (1995).

[5] A. Noreyan A, J. G. Amar, I. Marinescu, Molecular dynamics simulations of nanoindentation of beta-SiC with diamond indenter. Mater. Sci. Eng. B 117 (2005) 235-40

[6] I. Szlufarska, R. K. Kalia, A. Nakano, P. Vashishta, Atomistic mechanisms of amorphization during nanoindentation of SiC: a molecular dynamics study. Phys. Rev. B 71 (2005) 174113

[7] M. Mishra, I. Szlufarska, Possibility of high-pressure transformation during nanoindentation of SiC. Acta Mater. 57 (2009) 6156-65

[8] J-L. Demenet et al, Dislocations in 4H- and 3C-SiC single crystals in the brittle regime Phys. Status Solidi c10 (2013) 64-7

[9] X. Zhao et al, Onset plastic deformation and cracking behaviour of silicon carbide under contact load at room temperature J. Am. Ceram. Soc. 94 (2011) 3509-14 
[10] S. Goel, X. Luo, R. L. Reuben, Shear instability of nanocrystalline silicon carbide during nanometric cutting Appl. Phys. Lett. 100 (2012) 231902

[11] M. Mishra, I. Szlufarska, Dislocation controlled wear in single crystal silicon carbide J. Mater. Sci. 48 (2013) 1593-603

[12] P. Erhart, K. Albe, Analytical potential for atomistic simulations of silicon, carbon, and silicon carbide. Physical Review B, 71(3) (2005) 035211

[13] Tersoff, J., Chemical order in amorphous silicon carbide. Physical Review B, 49 (23) (1994) 16349

[14] A. Stukowski, K. Albe, Extracting dislocations and non-dislocation crystal defects from atomistic simulation data. Modelling Simul. Mater. Sci. Eng. 18 (2010) 085001

[15] S. Z. Chavoshi, S. Goel, X. Luo, Molecular dynamics simulation investigation on plastic flow behaviour of silicon during nanometric cutting. Modelling Simul. Mater. Sci. Eng. 24 (2016) 015002

[16] S. Z. Chavoshi, S. Goel, X. Luo, Influence of temperature on the anisotropic cutting behaviour of single crystal silicon: A molecular dynamics simulation investigation. Submitted for publication

[17] S. Z. Chavoshi, X. Luo, Defect mediated plasticity in silicon during nanometric cutting: A molecular dynamics simulation study. Submitted for publication

[18] S. Z. Chavoshi, X. Luo, An atomistic simulation investigation on chip related phenomena in nanometric cutting of single crystal silicon at elevated temperatures. Computational Materials Science, (2015) doi:10.1016/j.commatsci.2015.11.027

[19] S. Goel, X. Luo, A. Agrawal, R. L. Reuben, Diamond machining of silicon: A review of advances in molecular dynamics simulation, International Journal of Machine Tools \& Manufacture 88 (2015) 131-164 
[20] L. Pastewka, A. Klemenz, P. Gumbsch, M. Moseler, Screened empirical bond-order potentials for Si-C. Phys. Rev. B 87 (2013) 205410

[21] S. Plimpton, Fast parallel algorithms for short-range molecular-dynamics. Journal of computational physics, 117 (1) (1995) 1-19

[22] A. Stukowski, Visualization and analysis of atomistic simulation data with OVITO-the Open Visualization Tool Modelling Simul. Mater. Sci. Eng. 18 (2010), 015012

[23] Z. Li, R. C. Bradt, Thermal expansion of the cubic (3C) polytype of SiC. Journal of Materials Science 21 (1986) 4366-4368

[24] R. Hull, Properties of Crystalline Silicon. INSPEC publication (1999)

[25] W. R. L. Lambrecht, B. Segall, M. Methfessel, M. van Schilfgaarde, Calculated elastic constants and deformation potentials of cubic SiC. Phys. Rev. B 44 (1991) 3685

[26] H. V. Swygenhoven, P. M. Derlet, A. G. Frøseth, Stacking fault energies and slip in nanocrystalline metals. Nature Mater. 3, (2004) 399-403

[27] R. Peierls, The size of a dislocation. Proc. Phys. Soc. 52 (1940) 34-37

[28] F. R. N. Nabarro, Dislocations in a simple cubic lattice. Proc. Phys. Soc. 59 (1947) 256-272

[29] P. Vashishta, R. K. Kalia, A. Nakano, J. P. Rino, Interaction potential for silicon carbide: A molecular dynamics study of elastic constants and vibrational density of states for crystalline and amorphous silicon carbide. Journal of applied physics 101 (2007) 103515

[30] T. Thomas, D. Pandey, U. V. Waghmare, Soft modes at the stacking faults in SiC crystals: First-principles calculations. Physical Review B 77, 121203 (R) (2008)

[31] G. Cheng, T-H Chang, Qi. Qin, H. Huang, Y. Zhu, Mechanical Properties of Silicon Carbide Nanowires: Effect of Size- Dependent Defect Density. Nano Lett. 2014, 14, 754-758 
[32] Y. Umeno, K. Yagi, H. Nagasawa, ab initio density functional theory calculation of stacking fault energy and stress in 3C-SiC. Phys. Status Solidi B 249 (6) (2012) 1229-1234

[33] M. Mishra, I. Szlufarska, Dislocation controlled wear in single crystal silicon carbide. J Mater Sci 48 (2013) 1593-1603

[34] Y. Umeno, Y. Kinoshita, T. Kitamura, Ab initio DFT simulation of ideal shear deformation of SiC polytypes. Modelling Simul. Mater. Sci. Eng. 1527 (2007)

[35] L. Kubin, Dislocations, Mesoscale Simulations and Plastic Flow. Oxford publication, ISBN-13: $9780198525011(2013)$

[36] A. George, Plastic deformation of semiconductors: some recent advances and persistent challenges. Materials Science and Engineering A233 (1997) 88-102

[37] H. P. Chen, R. K. Kalia, A. Nakano, P. Vashishta, I. Szlufarska, Multimillion-atom nanoindentation simulation of crystalline silicon carbide: Orientation dependence and anisotropic pileup. Journal of Applied Physics 102 (6), 063514

[38] A. Boulle, D. Chaussende, F. Pecqueux, F. Conchon, L. Latu-Romain, O. Masson, Determination of stacking fault densities in 3C-SiC crystals by diffuse X-ray scattering. Phys. Status Solidi A, 204 (2007) 2528-2534

[39] S. Goel et al, Anisotropy of single-crystal 3C-SiC during nanometric cutting. Modelling Simul. Mater. Sci. Eng. 21 (2013) 065004

[40] M. Mishra, I. Szlufarska, Possibility of high-pressure transformation during nanoindentation of SiC. Acta Materialia 57 (2009) 6156-6165

[41] J. Patten, W. I. Gao, K. Yasuto, Ductile Regime Nanomachining of Single-Crystal Silicon Carbide. J Manuf Sci Eng-Trans ASME, 127 (2005), p. 522 
[42] V. I. Yamakov, D. H. Warner, R. J.Zamora, E. Saether, W. A. Curtin, E. H .Glaessgen, Investigation of crack tip dislocation emission in aluminum using multiscale molecular dynamics simulation and continuum modelling. Journal of the Mechanics and Physics of Solids 65(2014) 3553

[43] Y. Sun, S. Izumi, S. Sakai, K. Yagi, H. Nagasawa, Core element effects on dislocation nucleation in 3C-SiC: Reaction pathway analysis. Computational Materials Science 79 (2013) 216222

[44] J. P. Hirth and J. Lothe, Theory of Dislocations, John Wiley and Sons Inc, New York (1991)

[45] J. L. Demenet, M. H. Hong, P. Pirouz, Plastic behaviour of 4H-SiC single crystals deformed at low strain rates. Scripta Mater. 43, 865 (2000).

[46] L. Pizzagalli, Stability and mobility of screw dislocations in $4 \mathrm{H}, 2 \mathrm{H}$ and $3 \mathrm{C}$ silicon carbide. Acta Materialia 78 (2014) 236-244

[47] J. H. Lee et al., Direct observation of Lomer-Cottrell Locks during strain hardening in nanocrystalline nickel by in situ TEM. Scientific Reports 3 (2013) 1061

[48] V. Yamakov, D. Wolf, S. R. Phillpot, A. K. Mukherjee, H. Gleiter, Deformation-mechanism map for nanocrystalline metals by molecular-dynamics simulation. Nature Mater. 3, 43-47 (2004)

[49] V. Bulatov, F. Abraham, L. Kubin, B. Devincre, S. Yip, Connecting atomistic and mesoscale simulations of crystal plasticity. Nature, 391 (1998) 669

[50] S. Z. Chavoshi, X. Luo, An atomistic simulation study on the hot nanometric cutting of single crystal 3C-SiC. Submitted for publication

[51] P. K. Sitch, R. Jones, S. Öberg, M. I. Heggie, Ab initio investigation of the dislocation structure and activation energy for dislocation motion in silicon carbide. Phys. Rev. B 52 (1995) 4951 
[52] J. Li, W. Cai, J. Chang, S. Yip, Commentary on Atomistic Simulations of Materials Strength and Deformation: Prospects for Mechanistic Insights. ISMS-2, A (2001) 220-233

[53] S. P. Coleman, D. E. Spearot, L. Capolungo, Virtual diffraction analysis of Ni [010] symmetric tilt grain boundaries. Modelling and Simulation in Materials Science and Engineering, 21 (2013) 055020

[54] D. Huang, Y. Ikuhara, M. Narisawa, Kiyohito Okamura, Characterization of b-Silicon Carbide Powders Synthesized by the Carbothermal Reduction of Silicon Carbide Precursors. J. Am. Ceram. Soc., 81 (12) 3173-76 (1998)

[55] M. Yoshida, A. Onodera, M. Ueno, K. Takemura, O. Shimomura, Pressure-induced phase transition in SiC. Phys Rev B, 48 (1993), 10587

[56] A. Noreyan, J. G. Amar, I. Marinescu, Molecular dynamics simulations of nanoindentation of $\beta$-SiC with diamond indenter. Materials Science and Engineering B 117 (2005) 235-240

[57] G. Xiao, S. To, G. Zhang, The mechanism of ductile deformation in ductile regime machining of 6H-SiC. Computational Materials Science 98 (2015) 178-188

[58] S. Goel, X. Luo, A. Agrawal, R. L. Reuben, Diamond machining of silicon: A review of advances in molecular dynamics simulation, International Journal of Machine Tools \& Manufacture 88 (2015) 131-164 
